\title{
Communication and influence
}

\author{
Antoni Calvó-Armengol \\ ICREA, Universitat Autònoma de Barcelona and Barcelona GSE
}

JoAN DE MARTí

Departament d'Economia i Empresa, Universitat Pompeu Fabra and Barcelona GSE

Andrea Prat

Columbia Business School and Department of Economics, Columbia University

\begin{abstract}
We study the information flows that arise among a set of agents with local knowledge and directed payoff interactions, which differ among pairs of agents. First, we study the equilibrium of a game where, before making decisions, agents can invest in pairwise active communication (speaking) and pairwise passive communication (listening). This leads to a full characterization of information and influence flows. Second, we show that when the coordination motive dominates the adaptation motive, the influence of an agent on all his peers is approximately proportional to his eigenvector centrality. Third, we use our results to explain organizational phenomena such as the emergence of work "cliques," the adoption of human resources practices that foster communication (especially active communication), and the discrepancy between formal hierarchy and actual influence. KEYwords. Team theory, organizations, communication, invariant method.
\end{abstract}

JEL CLASSIFICATION. D83, D85.

\section{INTRODUCTION}

Communication is one of the defining characteristics of humans. A large part of our day is spent on various media, ranging from having informal conversations to writing

Joan de Martí: joan.demarti@upf .edu

Andrea Prat: andrea.prat@columbia.edu

Toni passed away in November 2007. We miss his friendship, energy, and talents. While the ideas and the results contained in this paper are due to the three authors, only de Martí and Prat are responsible for any remaining errors or omissions. A previous version of this paper was circulated under the title "Endogenous communication in complex organizations." We thank Jose Apesteguia, Can Celiktemur, Wouter Dessein, Jan Eeckhout, Luis Garicano, Bob Gibbons, Christian Hellwig, Rachel Kranton, David Myatt, Ignacio Palacios-Huerta, Alessandro Pavan, Daniel Rappoport, Karl Schlag, Juuso Välimäki, Fernando VegaRedondo, Yves Zenou, a co-editor, and three anonymous referees. We are also grateful to audiences at the University of Chicago, Columbia University, Duke University, MIT Sloan, Northwestern University, EUI (Florence), Universitat Pompeu Fabra, Stockholm University, and at the conference on "Social Networks and Peer Effects: Theory and Applications" in honor of Toni (Universitat Autònoma de Barcelona), the European Summer Symposium in Economic Theory (Gerzensee), and the Workshop on Communication and Belief Manipulation (Paris School of Economics).

Copyright $\odot 2015$ Antoni Calvó-Armengol, Joan de Martí, and Andrea Prat. Licensed under the Creative Commons Attribution-NonCommercial License 3.0. Available at http: //econtheory . org.

DOI: $10.3982 / \mathrm{TE} 1468$ 
formal reports, from exchanging email messages to participating in social media. This is true in social contexts as well as in the workplace. Corporate leaders spend upwards of 80 percent of their work time on communication-centered activities (Mintzberg 1973, Bandiera et al. 2011)

The endogeneity of communication patterns should lie at the center of a theory of organization (Arrow 1974). We have some control of to whom we decide to speak, email, or telephone. As communication requires time, we are selective and instrumental in how much we invest in communicating with different agents. As Simon (1986) noted, "If we record the frequency of communication between different nodes, we [will] find that the pattern is not uniform but highly structured. In fact, the pattern of communication frequencies [should] reflect, approximately, the pattern of authority." The objective of this paper is to develop a model of endogenous costly communication and to use it to understand influence patterns.

The model can be sketched as follows. There are a number of agents who face local uncertainty (for simplicity, local states are assumed to be mutually independent). Each agent observes the realization of his local state and must take an action. The payoff of each agent depends on his local state, his own action, and the action of other agents. For every pair of agents, action interdependence is measured as a continuous intensity and it can be asymmetric (agent $A$ places more importance on coordinating with $B$ than with $C$ ) and directed (agent $A$ wants to coordinate with $B$ more than $B$ wants to coordinate with $A$ ).

Before choosing his action, an agent can engage in communication. He can inform other agents about his own state of the world and he can gather information about other agents' states of the world. ${ }^{1}$ Formally, the agent selects a vector of active communication intensities and a vector of passive communication intensities. The precision of the communication of one agent to another is then determined by how much the sender invests in active communication (talking) and how much the receiver invests in passive communication (listening). Both types of communication are costly, and the cost is an increasing and convex function of communication intensity. In this model, the intensity of communication and influence (how much an agent's state influences another agent's action) is represented by continuous variables. This allows us to study varying degrees of interpersonal ties, as suggested by the sociological literature (Granovetter 1973).

Our analysis is divided in three parts. We first characterize communication patterns and individual decisions in equilibrium. We show that games in the class we consider have a unique equilibrium in linear strategies, namely one where the action of each agent is a linear function of his own signal and the signal that he receives from other agents.

These games feature various levels of strategic interaction. Active communication, passive communication, and decision influence are strategic complements. Alice invests in talking to Bob because she hopes that Bob will invest in listening to her and will

\footnotetext{
${ }^{1}$ In our setup, which allows for one round of communication only, there is no loss of generality in assuming that communication only relates to the observed state of the world. Things would be different if we allowed for more than one round.
} 
use the information he has received to coordinate his action's with Alice's state, something that would benefit Alice. Bob is influenced by the signal he receives from Alice because he has invested in listening and he thinks that she has invested in active communication; hence, the signal should be valuable in coordinating his action with hers. With more than two agents, these interactions may be indirect. Alice and Carol are not interested in coordinating their actions per se, but they both want to coordinate with Bob. In equilibrium they might invest in communicating with each other, in the knowledge that Bob's action will be affected by their states.

One way to find the fixed point of this set of interactions is to proceed iteratively, at each step taking into account a higher level of interactions. Our equilibrium corresponds to the limit of this iterative process. In equilibrium, a simple linear relation describes active and passive communication from one agent to the other as a linear function of how much the latter is influenced by information he receives. In turn, influence coefficients are determined jointly for all agents as a linear system. This tractable equilibrium characterization is useful to draw lessons on patterns of communication and influence.

In the second part of the paper, we use the main characterization result to explore patterns of communication and influence. As one would expect, we can prove that communication flows and influence relations between two agents become stronger if these agents have stronger exogenous interaction ties. Less obvious are indirect effects, namely how communication between two agents changes when other interaction ties change. Suppose a subset of workers is bound by strong exogenous ties: in the labor sociology language, a work "clique" exists (Dalton 1959). We show the existence of an insularity effect, pushing the subset of workers to reduce their interest in talking to the outside world, and an opposite reinforcement effect, whereby outsiders are keen to understand what goes on in the subset. Active and passive communication intensities move in opposite directions. The overall effect on influence from the subset to the rest of the workers is positive at first when they have relatively weak ties, but it turns to negative when the ties become sufficiently strong.

In our noncooperative setup, communication and influence is generally inefficiently low. Agents do not internalize the benefit that investment in communication generates for other agents. This is true for both active and passive communication. However, players tend to underinvest, in relative terms, more in active communication than in passive communication. To see this, suppose that active and passive communication have the same cost. A planner would choose the same level of active and passive communication. Yet, with $n>2$, agents spend less on active communication. The benefit of listening is direct: the receiver incorporates the information in his decision-making. The benefit of speaking is indirect: the sender hopes that the receiver will incorporate the information he receives in his decision-making. We relate these results to the evidence on the effect on firm performance of introducing innovative human resources management (HRM) practices (Ichniowski et al. 1997).

In the third and last part of the paper, we ask which agents are most influential overall. We define the global influence of an agent as the aggregate effect that a marginal change in the agent's local state has on other agents' actions. In our setting, global 
influence is an equilibrium variable, which is, in turn, jointly determined by three other equilibrium phenomena: active communication, passive communication, and decision-making.

We uncover a connection between global influence and eigenvector centrality. In the quadratic setting we study, each agent cares about adaptation (fitting his action to his own local state) and coordination (fitting his action to other agents' actions). We show that as the coordination motive becomes more and more important, the vector of global influences defined above tends to the vector of eigenvector centralities computed on a primitive of the problem: the matrix of directed interaction payoffs. This can be seen as an approximation result. In a game where coordination is much more important than adaptation, the relative global influence of every agent is well approximated by his eigenvector centrality. Given the simplicity of eigenvector measures, our result is useful for characterizing the asymptotic properties of the games we study.

This result relates to Ballester et al. (2006). They analyze a class of complete information games with quadratic payoffs and pairwise dependent strategic complementarities, and they show that in the equilibrium of these games, the effort exerted by each agent strongly depends on his position in the network of relations. In particular, this effort is proportional to his Bonacich index, a centrality measure constructed by summing paths emanating from one agent (with a decay factor). In our game, an agent's influence can be expressed as a linear function of Bonacich centralities and this fact is used in the proof of Theorem $5 .^{2}$

We apply the asymptotic equivalence result to study influence in hierarchical structures. Weber's top-down view of optimal bureaucracies came into question when empirical studies started showing that in the presence of conflicts of interest, the most influential members of an organization are often found at mid-level rather than at the top. Armed with the asymptotic correspondence between influence and eigenvector centrality, we first show that the Weberian view holds in a regular hierarchy, namely one where exogenous interaction patterns repeat themselves at every level. In the equilibrium of our communication game, a superior and his subordinates invest in reciprocal communication. Because of the tree-like nature of the hierarchy, this implies that the global influence of an agent is greater, the higher the agent is in the organization. However, Weber's monotonicity breaks down when there are groups of agents with stronger ties. In that case, as Dalton predicted, the most influential members of the organization are the top people within their "clique." The complementarity between interaction, communication, and influence means that the head of the clique is more influential, not only toward his subordinates, but also toward his superior. This corresponds to Dalton's observation that the most influential agents are often low-level managers.

The rest of the paper is organized as follows. Following the literature review, Section 2 introduces the model. Section 3 presents the first main result of the paper: the equilibrium characterization theorem. Section 4 uses the characterization theorem for

\footnotetext{
${ }^{2}$ The technical connection between our two papers is discussed in detail in the remarks after Theorem 5 . The obvious difference is that in Ballester et al. (2006), there is no information asymmetry and, hence, communication, and that they do not discuss eigenvector centrality.
} 
comparative statics and welfare analysis, with an emphasis on the role of complementarities, both between communication and decisions, and between active and passive communication. Section 5 reports the second main result-the asymptotic equivalence between agent's influence and the eigenvector centrality—and applies it to Dalton's hierarchies. Section 6 concludes by suggesting future avenues of research. All proofs are given in the Appendix.

A supplementary file available on the journal website, http://econtheory.org/supp/ 1468/supplement.pdf, provides an array of robustness checks and additional results: alternative time line, equilibrium uniqueness, ban on communication along certain links, broadcasting (as opposed to pairwise communication), corner solutions, and additional comparative statics. These extensions are referenced in the text in informal terms and proven formally in the supplementary material.

\subsection{Literature review}

This paper relates to two strands of literature: organizational economics and the economics of networks.

In the first strand of literature, there are a number of papers that study endogenous communication in a variety of settings. Our approach to multiperson decision-making under asymmetric information, as well as our normal-quadratic formulation, is inspired by Marschak and Radner's (1972) team theory. Some recent papers (Dessein and Santos 2006, Alonso et al. 2008, Rantakari 2008, Dessein et al. 2013) explore decentralized decision-making within organizations. Besides sharing their normal-quadratic setup, we are also interested in the trade-off between adaptation and coordination. We are particularly close to three models of endogenous costly communication between two agents.

In Dessein and Santos (2006), an agent can send a signal about his local state to the other agent, and the precision of the signal is endogenous. ${ }^{3}$ They show the existence of complementarities between communication, adaptation, and job description: in particular, when communication costs decrease, the organization is more likely to adopt a new set of organizational practices that include broader tasks and more adaptation.

Dewatripont and Tirole (2005) analyze a model of endogenous costly communication between a sender and a receiver. As in our model, both active and passive communication are endogenous and costly, and there are positive externalities (it takes two to communicate). Dewatripont and Tirole's communication model has a number of features that are absent here, such as the presence of signaling and the possibility of sending "cues," i.e., information about the sender's credibility.

Van Zandt (2004) develops a model of endogenous costly communication where several agents can transmit information at the same time. This leads to screening costs on the part of receivers and the potential for "information overload." Van Zandt examines

\footnotetext{
${ }^{3}$ One technical difference is that Dessein and Santos' (2006) signals are either fully informative or uninformative, and precision is defined as the probability that the signal is informative. Here, instead, signals are normally distributed and the precision is the reciprocal of the variance.
} 
possible mechanisms for reducing overload-an important problem in modern organizations. Our paper abstracts from information overload by assuming that receivers do not face a screening problem (they can always choose not to listen to a particular sender).

Our contribution with respect to these three papers is to extend endogenous communication to complex architectures. We discuss an array of phenomena that have limited or no meaning with only two agents, such as cliques, global influence, indirect effects, the opportunity to encourage active communication, etc.

Following the seminal work of Radner (1993), the literature of organizational economics has also studied the role of networks in minimizing human limitations in information processing. The works of Bolton and Dewatripont (1994), Van Zandt (1999a), Garicano (2000), Guimerà et al. (2002), and Dodds et al. (2003) highlight the importance of hierarchies, and more general network structures, to diminish the costs related to processing information that flows through the network of contacts. This literature is surveyed by Van Zandt (1999b) and Ioannides (2012). Our work is complementary to this one, and analyzes how individual payoff complementarities shape both the network structure of communication and the equilibrium actions.

Crémer et al. (2007) formalize Arrow's (1974) idea of coding: the medium of communication used by a group of people (the organizational language) is endogenous and it determines communication costs. For analytical tractability, in the present model the communication medium is not modeled explicitly, but it is represented by a communication cost function. ${ }^{4}$

Related work can also be found in political economy. Dewan and Myatt (2008) analyze the role of communication in the interplay of leaders and activists in political parties. Leaders are heterogeneous in two different skills: their ability to interpret which is the correct policy to promote, and the clarity of communication of their ideas to the activists. Activists seek to advocate for the correct policy by listening with different intensities to the party leaders. The authors show that, generally, clarity in communication is the leaders' ability to induce higher influence on activists' opinions. Dewan and Myatt's interpretation of communication is close to the one we propose in our work: in a Bayesian game with quadratic payoff functions and normally distributed signals that represent the messages sent and received, agents can affect the precision of these signals. Alternatively, the communication protocols and, therefore, the strategic effects of communication are different in the two models, as well as the questions that are analyzed.

In the second strand of literature-network economics-the closest contribution is Calvó-Armengol and de Martí Beltran (2009), who consider a normal-quadratic teamtheoretical setup and study the effect of communication among agents. The authors provide a full characterization of the decision functions and the equilibrium payoffs given a communication structure. Calvó-Armengol and de Martí Beltran also study what the best communication structure is when the overall number of links among agents is

\footnotetext{
${ }^{4}$ Crémer (1993) and Prat (2002) study costly endogenous information collection in a team-theoretic setting. Hellwig and Veldkamp (2009) examine optimal information choices in a strategic setting. The present paper is complementary in that it endogenizes communication rather than information collection.
} 
bounded: they provide sufficient conditions for the optimal communication network to be a star or the maximum aggregate span network. However, the only choice between two nodes is no communication or full communication, so the kind of communication intensity analysis that we perform here is absent.

This paper also adopts a normal-quadratic specification, close to the one in CalvóArmengol and de Martí Beltran. The key innovation here is, of course, that communication is endogenous. We also move away from a team-theoretical framework, we introduce the idea of communication intensity, and we distinguish between active and passive communication. ${ }^{5}$

Our eigenvector centrality result is related to three papers. Golub and Jackson (2010) study a learning model where agents communicate on a network and process signals they receive naively. They show that when beliefs converge, the consensus belief is a weighted average of agents' initial beliefs and the weights, which correspond to a measure of global influence, are given by a principal eigenvector of the social network matrix. Elliott and Golub (2013) study a setting where agents can exert costly effort to generate nonrival, heterogeneous benefits for other agents and characterize Lindahl outcomes as the eigenvector centrality action profiles. Golub and Lever (2010) study how eigenvector centrality of agents in a network changes when initially disconnected groups begin interacting with each other via a new bridging link. Weak intergroup links can have arbitrarily large effects on the distribution of centrality.

Hagenbach and Koessler (2010) and Galeotti et al. (2013) also consider, as we do, strategic endogenous communication in a network game. However, their focus is on costless, nonverifiable information (cheap talk) when agents may have biases as in Crawford and Sobel (1982) (see Sobel 2013 for a survey). Our setup is different in that we focus on costly and verifiable information. The kind of issues we address are thus entirely different (and complementary). ${ }^{6}$

With regard to the literature on the formation of (communication) networks, Bloch and Dutta (2009) study the creation of communication networks with endogenous link strength. In their model, agents have a fixed resource, for example, time, and have to decide how to allocate it to create connections with others. The benefits of a connection depends on the decisions of both agents involved in it. Furthermore, in the spirit of the connections model introduced in Jackson and Wolinsky (1996), an agent obtains benefits of indirect connections through the more reliable path connecting them with each one of the agents in the society.

Rogers (2008) analyzes another network formation game in which all agents have a limited resource available to spend building links with the rest of agents, but differs with

\footnotetext{
${ }^{5}$ The literature of information sharing in oligopoly has also considered a normal-quadratic setup. See, for example, Vives (1984), Gal-Or (1985, 1986), and Raith (1996). Vives (1999) surveys this literature. While the setup in these papers bears some resemblance to our paper, there are several differences in the analysis, both because communication is public and, hence, there is no network component, and because the focus is quite different (industry competition rather than organization economics).

${ }^{6} \mathrm{~A}$ point of overlap with Hagenbach and Koessler (2010) is their result that when information is fully verifiable, agents will want to communicate all they know. This corresponds to our setup when communication costs go to zero.
} 
the work of Bloch and Dutta in the structure of benefits. In Rogers (2008), the utility of an agent depends on the utility of each other agent with whom he is directly connected. This recursive definition of utilities generates indirect effects that spread through indirect connections of any length. The author analyzes two games: one in which the dependency expresses that each agent gives utility to his connections; another one in which the dependency expresses that each agent receives utility from his connections. In both cases, the Nash equilibria are characterized.

Our paper is also linked with the growing literature on games played in a network, in which players' payoffs are intimately related to the geometry of relations among them. ${ }^{7}$ Ballester et al. (2006) has already been cited in the Introduction. Galeotti and Goyal (2010) model both the network formation process and the play of a game that depends on the network formed. The authors study a game in which payoffs depend on the (costly) information they acquire and gather from their neighbors in a network of relations. The analysis of this game in a fixed network is performed in Bramoullé and Kranton (2007), in which a set of varied possible equilibria are presented. The novelty in Goyal and Galeotti is that they allow agents to choose their connections. They show that the introduction of endogenous network structures induces a simpler core-periphery structure in the equilibrium formed. In particular, equilibrium networks show a coreperiphery pattern in which a set of few individuals are highly connected with a high number of poorly connected agents. While their setup is different from ours, we share Goyal and Galeotti's goal of studying a two-stage game, in which the first stage involves investment in links (in our case, communication precision) and the second stage involves play on the basis of those links. Cabrales et al. (2011) study a network game where network formation and productive efforts are selected simultaneously, and show that there exist two stable interior Pareto-rankable equilibria. The setup we study could have another pure-strategy equilibrium characterized by a coordination failure, but we can rule it out by assuming that an infinitesimal amount of information transmission occurs even if agents do not invest in communication at all (see the discussion of uniqueness after Theorem 1).

\section{MODEL}

Consider a set of $n$ agents. Agent $i$ faces a local state of the world

$$
\theta_{i} \sim \mathcal{N}\left(0,1 / s_{i}\right)
$$

where $s_{i}$ denotes the precision of $\theta_{i}$, i.e., $s_{i}=1 / \operatorname{Var}\left(\theta_{i}\right)$. The local states of different agents are mutually independent. Agent $i$ observes only $\theta_{i}$.

\footnotetext{
${ }^{7}$ We analyze an incomplete information game played in a network. However, as usual in the literature, we assume full knowledge by all players on the realized network structure. For some facts about network games with incomplete information on the network structure, we refer the interested reader to Galeotti et al. (2010).
} 
All agents engage in pairwise communication activity. ${ }^{8}$ Agent $i$ receives message $y_{i j}$ from agent $j$, such that

$$
y_{i j}=\theta_{j}+\varepsilon_{i j}+\eta_{i j},
$$

where $\varepsilon_{i j}$ and $\eta_{i j}$ are two normally distributed noise terms

$$
\begin{aligned}
\varepsilon_{i j} & \sim \mathcal{N}\left(0,1 / r_{i j}\right) \\
\eta_{i j} & \sim \mathcal{N}\left(0,1 / p_{i j}\right),
\end{aligned}
$$

and $r_{i j}$ (resp. $p_{i j}$ ) is the precision of $\varepsilon_{i j}$ (resp. $\eta_{i j}$ ). We interpret $\varepsilon_{i j}$ as the noise associated with active communication (preparing a presentation, writing a report, hosting a visit, hosting a liaison officer) and interpret $\eta_{i j}$ as the noise associated with passive communication (listening to a presentation, reading a report, visiting a plant, appointing a liaison officer). All the noise terms are stochastically independent from each other and from the local states.

Agent $i$ chooses how much to invest in speaking with and listening to other players. Namely, he selects among the following choices.

- The precision of the active communication part of all the signals he sends, $\left(r_{j i}\right)_{j \neq i}$, for which he incurs cost $k_{r}^{2} \sum_{j \neq i} r_{j i}$, where $k_{r} \geq 0$ is a parameter.

- The precision of the passive communication part of all the signals he receives, $\left(p_{i j}\right)_{j \neq i}$, for which he incurs cost $k_{p}^{2} \sum_{j \neq i} p_{i j}$, where $k_{p} \geq 0$ is a parameter ( $p$ is mnemonic for passive).

Thus, communication technology and communication costs are separable, both between active and passive communication and across agents. The absence of mechanical complementarities will allow us to highlight the presence of strategic complementaries, both between active and passive communication and across agents, which we will explore in detail in Section $4 .^{9}$

We also assume that each precision term is bounded below by a very small number $\xi: r_{j i} \geq \xi, p_{i j} \geq \xi{ }^{10}$ This avoids dominated equilibria where $i$ does not speak to $j$ because he does not expect $j$ to listen and vice versa. ${ }^{11}$

After observing the local state $\theta_{i}$ and the vector of signals $\left(y_{i j}\right)_{j \neq i}$, agent $i$ chooses an action $a_{i} \in(-\infty, \infty)$.

\footnotetext{
${ }^{8}$ Here we assume that agents can, in principle, communicate with all other agents. The analysis can be extended to situations where there are exogenous barriers to communication between certain pairs of agents. See Section S3 of the supplementary material for an example.

${ }^{9}$ To keep notation simpler, we assume that the cost parameters, $k_{p}$ and $k_{r}$, are the same for all agents and all pairs. A straightforward extension is to let the cost parameters depend on the identity of the agent incurring the cost or even on the identity of both sender and receiver. Theorem 1 would hold with small modifications.

${ }^{10}$ We hold $\xi$ fixed and take all other parameters to be such that nonzero equilibria satisfying these constraints exist.

${ }^{11}$ A natural question is whether, in this model, speaking and listening are strategic complements. The answer to this question is not straightforward at this stage and we postpone it to the discussion that follows the main result, Theorem 1.
} 
This setup contains two implicit assumptions. The first one, which was already discussed in the Introduction, is that when engaging in active communication, the agents cannot manipulate the average of the signals they send (the report may be more or less clear, but it cannot contain lies; the liaison officer cannot be bribed). The second assumption is that agents do not observe the communication intensities chosen by other agents directly (i.e., the agent does not see how much effort the others put into writing their reports or into reading his reports; the opportunity cost of sending/hosting a particular liaison officer is unobservable). If precisions were observable, each agent would have at his disposal a vector of noiseless continuous signals, which he could use to communicate his state perfectly.

The payoff of agent $i$ is quadratic,

$$
u_{i}=-\left(d_{i i}\left(a_{i}-\theta_{i}\right)^{2}+\sum_{j \neq i} d_{i j}\left(a_{i}-a_{j}\right)^{2}+k_{r}^{2} \sum_{j \neq i} r_{j i}+k_{p}^{2} \sum_{j \neq i} p_{i j}\right),
$$

where the term $d_{i i}$ measures the adaptation motive, i.e., the importance of tailoring $i$ 's action to the local state, and the term $d_{i j}$ represents the coordination motive, namely the interaction between the action taken by agent $i$ and the action taken by agent $j$. For the rest of the paper, we assume that the interaction terms are positive $\left(d_{i j} \geq 0\right.$ for all $i$ and all $j) .12$

For now, we study a game where agents invest in communication before observing their local state. Namely, the time line comprises the following steps.

1. Agents simultaneously select their active and passive communication intensity vectors $\left(r_{j i}\right)_{j \neq i}$ and $\left(p_{i j}\right)_{j \neq i}$.

2. Agents observe their local state of the world $\theta_{i}$.

3. Agents receive signals from other agents $\left(y_{i j}\right)_{j \neq i}$.

4. Agents select their actions $a_{i}$.

We refer to this game as $\Gamma(\mathbf{D}, \mathbf{k}, \mathbf{s})$, where $\mathbf{D}=\left(d_{i j}\right)_{i, j}, \mathbf{k}=\left(k_{r}, k_{p}\right)$, and $\mathbf{s}=\left(s_{i}\right)_{i}$.

One can think of a different timing in which agents invest in communication after observing their local state, in which case stages 1 and 2 in the time line above are inverted. The timing choice depends on whether one thinks of communication investment as long term or short term. Under this alternative timing, the investment in active and passive communication may depend on agents' local states. For the rest, the game is identical and we denote it with $\Gamma_{\theta}(\mathbf{D}, \mathbf{k}, \mathbf{s})$. The notation is mnemonic for the fact that communication intensities are chosen after the $\theta$ is observed. In the next section, we will discuss an equivalence result between these two versions of the game.

The two main assumptions of our model-quadratic payoffs and normal distribution of signals-are standard in the organizational economics literature inspired by team theory (Marschak and Radner 1972). ${ }^{13}$ They are usually justified as limiting results.

\footnotetext{
${ }^{12}$ In our notation, whenever a variable has two agent indices, such as $y_{i j}$ or $d_{i j}$, the first index denotes the agent who is "directly affected," such as the receiver of a signal or the owner of the payoff.

${ }^{13}$ See Garicano and Prat (2013) for a survey.
} 
Quadratic payoffs can be thought of as second-order local approximations of any differentiable utility function. ${ }^{14}$ In our case, the normal distribution of signals can be seen, with the use of the central limit theorem, as the limiting distribution in a scenario where information comes from many small and independent binary pieces of information that are transmitted satisfactorily, or otherwise are meaningless, with some probability and where the receiver averages all the pieces he receives from a sender.

\section{COMMUNICATION AND DECISIONS IN EQUILIBRIUM}

In this section, we study how agents invest in communication and make decisions in equilibrium. Before stating the formal result, it is useful to provide an informal discussion of the equilibrium structure. As mentioned in the Introduction, this game has a complex array of interaction effects.

The game is solved by backward induction. In the second stage, agents know their own communication intensities and have a conjecture about other agents' intensities. On the basis of this, they use the signals they receive to choose their actions. Given the linear-quadratic structure of the game, the optimal action for agent $i$ is given by

$$
D_{i} a_{i}^{*}\left(\mathbf{y}_{i}\right)=d_{i i} \theta_{i}+\sum_{j \neq i} d_{i j} \mathbb{E}\left[a_{j}^{*} \mid \mathbf{y}_{i}\right] .
$$

In what follows, we denote the sum of his interaction parameters for agent $i$ with

$$
D_{i}=\sum_{j=1}^{n} d_{i j}
$$

and denote the normalized interaction parameter between $i$ and $j$ as

$$
\omega_{i j}=\frac{d_{i j}}{D_{i}} .
$$

The first-order condition (2) is thus

$$
a_{i}^{*}\left(\mathbf{y}_{i}\right)=\omega_{i i} \theta_{i}+\sum_{j \neq i} \omega_{i j} \mathbb{E}\left[a_{j}^{*} \mid \mathbf{y}_{i}\right],
$$

which contains a directly observable term $\left(\theta_{i}\right)$ and a first-order expectation (the other agents' actions).

Combining the first-order conditions of the form (3) generates iterated expectations. For instance, one additional round yields

$$
a_{i}^{*}\left(\mathbf{y}_{i}\right)=\omega_{i i} \theta_{i}+\sum_{j \neq i} \omega_{i j} \mathbb{E}\left[\omega_{j j} \theta_{j}+\sum_{k \neq j} \omega_{j k} \mathbb{E}\left[a_{k}^{*} \mid \mathbf{y}_{j}\right] \mid \mathbf{y}_{i}\right],
$$

\footnotetext{
${ }^{14}$ While we focus in the paper on a specific form of quadratic games, with a particular adaptationcoordination structure, our results can easily be extended to any quadratic utility function where, as below in text, the cross-effects in individual actions would determine the interaction matrix we use.
} 
which contains a directly observable term $\left(\theta_{i}\right)$, a first-order expectation on the other agents' local states, and a second-order expectation (the other agents' actions). The good news is that expectations on other agents' local states can be represented in a linear form, for any order. For instance, the first-order expectation term of $i$ on $j$ 's state is

$$
i \rightarrow j: \quad \omega_{i j} \mathbb{E}\left[\omega_{j j} \theta_{j} \mid \mathbf{y}_{i}\right]=\underbrace{\omega_{i j} \omega_{j j}}_{\text {second-order coord. concern }} \times \underbrace{\frac{p_{i j} r_{i j}}{p_{i j} s_{j}+r_{i j} s_{j}+p_{i j} r_{i j}}}_{\text {information extraction }} \times y_{i j} .
$$

The information extraction ratio is the classical formula for the expected value of an unobserved normally distributed variable (the local state $\theta_{j}$ in this case) given an observed normally distributed signal (the signal from $j$ to $i$, i.e., $y_{i j}$ ). In variance terms, it corresponds to

$$
\frac{\frac{1}{s_{j}}}{\frac{1}{s_{j}}+\frac{1}{p_{i j}}+\frac{1}{r_{i j}}} .
$$

The ratio is decreasing in the precision of the local state, $s_{j}$, and increasing in the precision of the signal, the sum of the speaking investment $r_{i j}$, and the listening investment $p_{i j}$.

In the next round, the second-order expectation term of $i$ on $k$ 's state, mediated by j's signal, is

$$
\begin{aligned}
i \rightarrow j \rightarrow k: & \omega_{i j} \omega_{j k} \mathbb{E}\left[\mathbb{E}\left[\omega_{k k} \theta_{k} \mid \mathbf{y}_{j}\right] \mid \mathbf{y}_{i}\right] \\
= & \underbrace{\omega_{i j} \omega_{j k} \omega_{k k}}_{\text {third order coord. concern }} \times \underbrace{\frac{p_{i j} r_{i j}}{p_{i j} s_{j}+r_{i j} s_{j}+p_{i j} r_{i j}} \frac{p_{j k} r_{j k}}{p_{j k} s_{k}+r_{j k} s_{k}+p_{j k} r_{j k}}}_{\text {information extraction about } k} \times y_{i k}
\end{aligned}
$$

and so on.

In the proof of Theorem 1, we show that expectations converge and we can always find an equilibrium of the form

$$
a_{i}^{*}\left(\mathbf{y}_{i}\right)=b_{i i} \theta_{i}+\sum_{j \neq i} b_{i j} y_{i j}
$$

Furthermore the $b$ coefficients are unique, and they can be characterized in a relatively simple way as a function of the communication intensities that are chosen in the first stage, the $p$ 's and the $r$ 's.

With these conditions in mind, we can now move to the first stage of the game, where agents make communication investments. Recall that agent $i$ 's loss, net of communication costs, is given by

$$
d_{i i}\left(a_{i}-\theta_{i}\right)^{2}+\sum_{j \neq i} d_{i j}\left(a_{i}-a_{j}\right)^{2} .
$$

If communication is poor, agent $i$ suffers in two ways: his own action $a_{i}$ contains an additional noise and the other agents' actions, the $a_{j}$ 's, contain additional noise. By (4), 
we can write

$$
\operatorname{Var}\left(a_{i}^{*}\right)=b_{i i}^{2} \operatorname{Var}\left(\theta_{i}\right)+\sum_{j \neq i} b_{i j}^{2} \operatorname{Var}\left(y_{i j}\right),
$$

which, combined with (5), implies that investment in passive communication $p_{i j}$ yields expected marginal benefit $D_{i} b_{i j}^{2}$ and investment in active communication $r_{j i}$ yields $d_{i j} b_{j i}^{2}$. Given our assumptions on communication costs, equilibrium communication intensities must be given by

$$
p_{i j}=\frac{\sqrt{D_{i}} b_{i j}}{k_{p}} \quad \text { and } \quad r_{j i}=\frac{\sqrt{d_{i j}} b_{j i}}{k_{r}} .
$$

Now, as shown in the proof, we can close the analysis by plugging equilibrium intensities in the second-stage decision coefficient $b$. To provide a complete characterization, we introduce one last piece of notation: for any pair of individuals $i$ and $j$, we define

$$
h_{i j}= \begin{cases}\omega_{j j} & \text { if } i=j \\ -s_{j}\left(\frac{k_{p}}{\sqrt{D_{i}}}+\frac{k_{r}}{\sqrt{d_{j i}}}\right) & \text { otherwise. }\end{cases}
$$

Also, define $\boldsymbol{\Omega}$ as a square matrix with $(i, j)$ entry equal to $\omega_{i j}$ and 0 on the diagonal. We can show (see the Appendix for a complete proof) the following theorem.

Theorem 1. For any $(\mathbf{D}, \mathbf{s})$, if $k_{r}$ and $k_{p}$ are sufficiently low, the game $\Gamma(\mathbf{D}, \mathbf{k}, \mathbf{s})$ has an equilibrium where the following statements hold:

(i) Decisions are given by

$$
\mathbf{b}_{\cdot j}=(\mathbf{I}-\mathbf{\Omega})^{-1} \cdot \mathbf{h}_{\cdot j} \quad \text { for all } j .
$$

(ii) Active communication is

$$
r_{i j}=\frac{\sqrt{d_{j i}} b_{i j}}{k_{r}} \quad \text { for all } i \neq j .
$$

(iii) Passive communication is

$$
p_{i j}=\frac{\sqrt{D_{i}} b_{i j}}{k_{p}} \quad \text { for all } i \neq j .
$$

The theorem offers a simple equilibrium characterization. Conditions (ii) and (iii) correspond to (6), and express each communication intensity as a linear function of just one decision coefficient. It requires that $k_{r}$ and $k_{p}$ are sufficiently low. If this condition fails, the lower-bound constraint on communication intensities may be violated. In that case, the equilibrium involves corner solutions, whereby some or all of the $p$ 's and $r$ 's are equal to the minimal value $\xi$. This possibility is explored in Section S5 of the supplementary material. However, for any $(\mathbf{D}, \mathbf{s})$, there always exist values of $k_{p}$ and $k_{r}$ that are sufficiently low to guarantee an interior solution.

Condition (i) is based on a characterization of $\mathbf{b}$ that is particularly tractable because (a) it does not depend on communication intensities (it only depends on primitives), 
(b) it can be split into $n$ systems of equations, one for each agent, (c) it is linear, and (d) it uses a coefficient matrix that is the same for all $n$ agents.

While system (i) does not contain $r$ and $p$, one should not think that it captures only decision considerations. As the presence of communication parameters $k_{r}$ and $k_{p}$ indicates, (i) also embodies communication considerations. Specifically, each subsystem in condition (i) determines all the coefficients $\left(b_{i j}\right)_{i=1, \ldots, n}$ in the linear decision function of agent $i$; each coefficient determined the effect of information originating from a certain agent $j$, namely, the signal $y_{i j}$ if $i \neq j$ and the local state $\theta_{j}$ if $i=j$.

The matrix $(\mathbf{I}-\boldsymbol{\Omega})^{-1}$ admits a simple interpretation. Since $\boldsymbol{\Omega}$ is a contraction (given its definition and the assumption that $d_{i i}>0$ for all $i$ 's), we can write

$$
(\mathbf{I}-\mathbf{\Omega})^{-1}=\mathbf{I}+\boldsymbol{\Omega}+\mathbf{\Omega}^{2}+\mathbf{\Omega}^{3}+\cdots=\sum_{l \geq 0} \boldsymbol{\Omega}^{l} .
$$

The entry $\omega_{i j}$ of $\boldsymbol{\Omega}^{l}$ is the sum of all products of normalized coordination concerns of all possible chains comprising exactly $l$ connected agents that start with agent $i$ and finish with agent $j$. Hence, the entry $\vartheta_{i j}$ of $(\mathbf{I}-\boldsymbol{\Omega})^{-1}$ is based on all product chains from $i$ to $j$, comprising any number of agents. The $\vartheta$ terms are always positive, and they measure the strength of direct and indirect coordination concerns between two agents. The matrix $(\mathbf{I}-\boldsymbol{\Omega})^{-1}$ is the same for all subsystems because the propagation of interaction effects across agents goes through the same payoff matrix: a change in any part of $j$ 's decision function affects $i$ through coefficient $d_{i j}$.

With this notation, the theorem says that the influence of agent $j$ over agent $i$ is determined as

$$
b_{i j}=\sum_{m=1}^{n} \vartheta_{i m} h_{m j}=\vartheta_{i j} \omega_{j j}-s_{j} \sum_{m \neq j} \vartheta_{i m}\left(\frac{k_{p}}{\sqrt{D_{m}}}+\frac{k_{r}}{\sqrt{d_{j m}}}\right) .
$$

The influence coefficient $b_{i j}$ has a positive term and $n-1$ negative terms. The positive term is larger when $\omega_{j j}=d_{j j} / D_{j}$ is large, namely, when the sending agent's adaptation concerns are large compared to his coordination concerns. That shows a natural effect: if $j$ 's adaptation concern is strong, his action is going to be close to his local, private information; knowing this, agent $i$ reacts by increasing his weight in the message he receives from $j$, and this reaction is stronger the more he cares to coordinate (directly or because other individuals he cares about want to coordinate with $j$ ) with $j$, as captured by $\vartheta_{i j}$.

Each negative term of the form $\vartheta_{i m} h_{m j}$ dampens the influence of $j$ over $i$ and relates to three factors: the predictability of $j$ 's environment $\left(s_{j}\right), i$ 's desire to coordinate with $m\left(\vartheta_{i m}\right)$, and the cost of communication relative to the desire for coordination $\left(k_{p} / \sqrt{D_{m}}+k_{r} / \sqrt{d_{j m}}\right)$, which will be discussed at length in the next section.

Regarding (ii) and (iii), there is an asymmetry between the two expressions: investment in active communication depends on one interaction parameter $\left(d_{j i}\right)$ while investment in passive communication depends on the sum of all parameters $\left(D_{i}\right)$. This asymmetry reflects a difference in the individual benefit of learning from others and the individual benefit of informing others. This strategic asymmetry between listening and speaking will be discussed at length in Section 4.2 . 
When communication cost vanishes ( $k_{p} \rightarrow 0, k_{r} \rightarrow 0$ ), the theorem predicts that all communication precisions grow unboundedly. The vector of actions tends to a simple limit:

$$
\mathbf{a}^{*}=(\mathbf{I}-\boldsymbol{\Omega})^{-1}\left(\begin{array}{c}
\omega_{11} \theta_{1} \\
\vdots \\
\omega_{n n} \theta_{n}
\end{array}\right) .
$$

The robustness of the result can be probed in a number of directions. First, we can ask what happens if, instead of assuming that communication investments are made before learning the local states, we assume the opposite. Recall that we defined $\Gamma_{\theta}(\mathbf{D}, \mathbf{k}, \mathbf{s})$ as a game that is identical to $\Gamma(\mathbf{D}, \mathbf{k}, \mathbf{s})$ except that the order of the first two stages is reversed. In $\Gamma_{\theta}$, agents invest after they observe their $\theta$. In general, we would expect the set of equilibria to be different because the incentive of agents to invest in active and passive communication may depend on the value of their local states. This, in turn, could create a signaling issue. However, we can prove that game $\Gamma_{\theta}(\mathbf{D}, \mathbf{k}, \mathbf{s})$ has a perfect Bayesian equilibrium that corresponds to the pure-strategy equilibrium of $\Gamma(\mathbf{D}, \mathbf{k}, \mathbf{s})$. The intuition for this result has to do with the assumption that payoff functions are quadratic. The choice of $a_{i}$ does, of course, depend on the value of $\theta_{i}$. However, the marginal benefit of additional information is the same whether the agent knows the actual value of his local state, as in $\Gamma_{\theta}$, or only its expected value, as in $\Gamma$. Strange signaling outcomes are also prevented by use of a probability distribution with a full support. This result is stated and proven in Section S1 of the supplementary material.

Second, is the equilibrium in Theorem 1 unique? First, the assumption that communication intensities are bounded below by $\xi$ rather than zero prevents the existence of equilibria based on pure miscoordination. Second, there could be nonlinear equilibria. In the equilibrium that we describe, the actions of agents are linear functions of the signals they receive. Within this class, it is immediate to see that our equilibrium is unique. This is a common assumption in the literature that uses the normal-quadratic approach, but while there are no known counterexamples, it is not obvious to show that only linear equilibria are possible (e.g., Angeletos and Pavan 2009, Dewan and Myatt 2008). While we cannot prove uniqueness in our game, we can prove uniqueness in a sequence of games whose limit is the game under consideration. In Section S2 of the supplementary material, we consider a truncated version of our game, where agents' action are bounded below and above. We show that this game has a unique equilibrium and that such equilibrium tends to ours as the bound goes to infinity.

Third, one may also think that in reality, communication can only occur between certain nodes. Namely, information transmission is impossible on certain edges of the graphs. Our equilibrium characterization can be modified to accommodate this additional constraint (Section S3 of the supplementary material).

Also note that a characterization similar to Theorem 1 obtains even under different assumptions on the structure of communication costs. For instance, rather than having pairwise communication, we could imagine that the agents "broadcast" their signals. Thus, each agent sustains the cost of active communication only once. This possibility is explored in Section S4 of the supplementary material. 


\section{Complementarities in COMmunication AND influence}

As shown in the equilibrium characterization result, there are strategic complementarities between active communication, passive communication, and decision-making that arise in models with three or more agents. This section builds on Theorem 1 to explore two aspects of these complementarities. It first studies indirect effects of the interaction parameters on communication and decisions, namely what happens to $b_{i j}, r_{i j}$, and $p_{i j}$ when $d_{i j}$ stays constant but other interaction parameters change. As we shall see, there are subtle nonmonotonic effects. The second part of the section focuses on inefficiencies arising from the complementarity between communication and decision-making. In particular, we will see that when there are three or more agents, underinvestment in active communication is particularly acute, and we will argue that in real organizations, this form of inefficiency is reduced by management practices that encourage active communication.

\subsection{Indirect effects}

It is easy to check that if there are only two players, comparative statics is relatively straightforward: an increase in the strength of the interaction parameters $d_{12}$ and $d_{21}$ leads to more investment in communication between the agents. This confirms previous results based on the presence of two agents only (Dewatripont and Tirole 2005, Dessein and Santos 2006).

However, as we mentioned earlier, how Alice influences Bob does not only depend on the direct interaction between Alice and Bob, but potentially on all other interactions. The analysis of indirect effects is less straightforward.

The following example, which provides a link to the sociology of labor, is the simplest way to illustrate the kind of nonmonotonic effects that can arise. Consider three agents with the interaction matrix

$$
D=\left[\begin{array}{lll}
d & q & \frac{1}{q} \\
q & d & \frac{1}{q} \\
1 & 1 & d
\end{array}\right] \quad \text { with } q \geq 1
$$

When $q=1$, the three agents are symmetric. As $q$ increases, a "clique" (to borrow a term used by labor sociologists (Dalton 1959)) forms between the first two agents with the exclusion of the third, the "outsider."

What happens when the exogenous link between agents 1 and 2 becomes stronger? As agents 1 and 2 care less about the outsider, there is a reduction in both communication by and influence of the outsider. The effect on communication and influence in the other direction is instead nonmonotonic. First, there is a clique insularity effect: agents 1 and 2 care less about the outsider and, hence, they invest less in talking to him: active communication variables $r_{13}$ and $r_{23}$ decrease (Figure 1(a)). Second, the outsider is affected by the clique reinforcement effect. As agents 1 and 2 communicate and coordinate more with each other, their mutual influence increases. The outsider realizes that agent 1's and agent 2's local states affect each other's actions and he becomes even more eager to learn them. He invests more in passive communication, at least for low values of $q$ (Figure 1(b)). When $q$ is sufficiently high, the insularity effect must dominate. As agents 


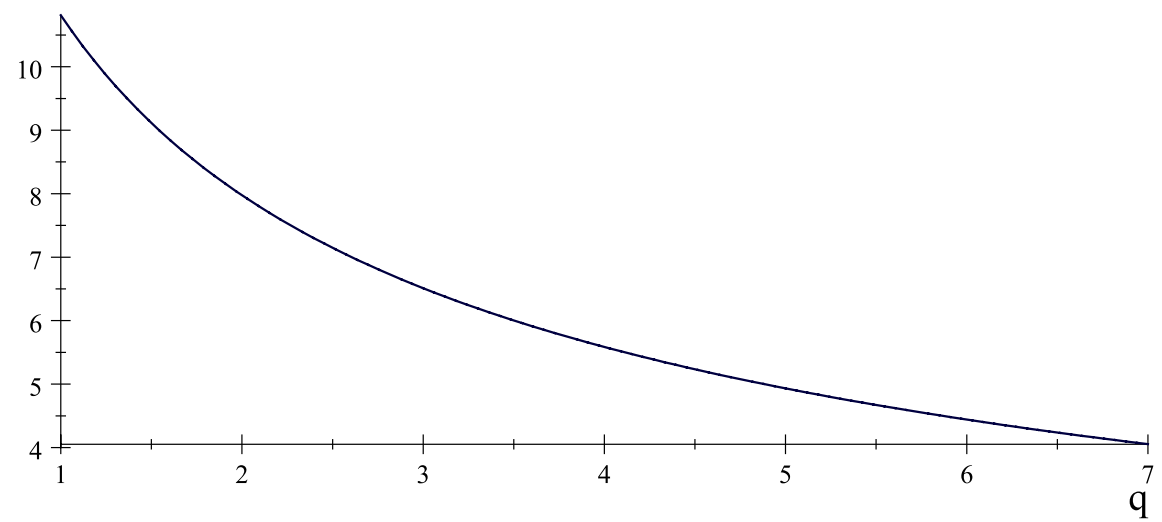

(a)

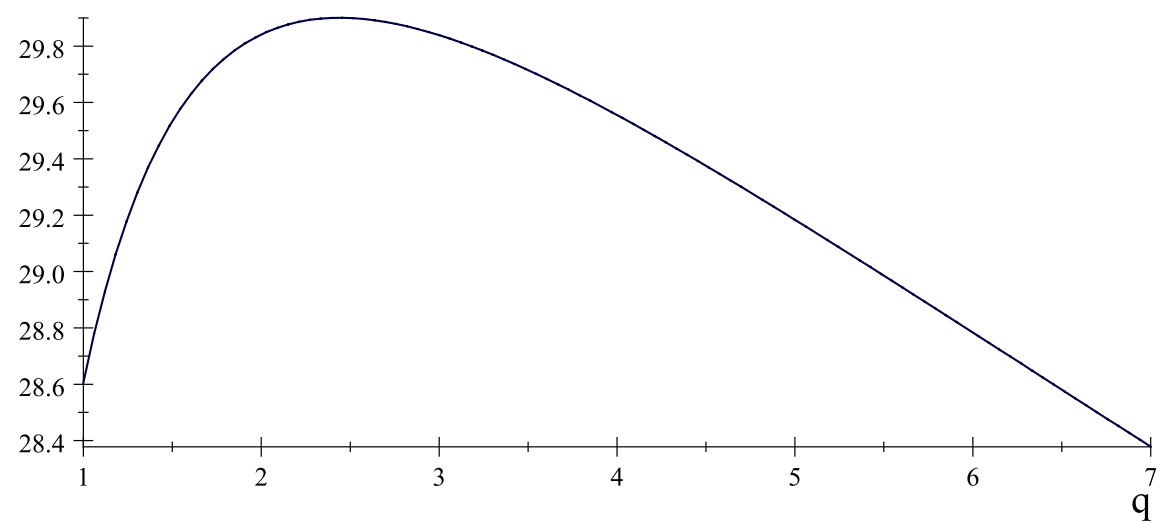

(b)

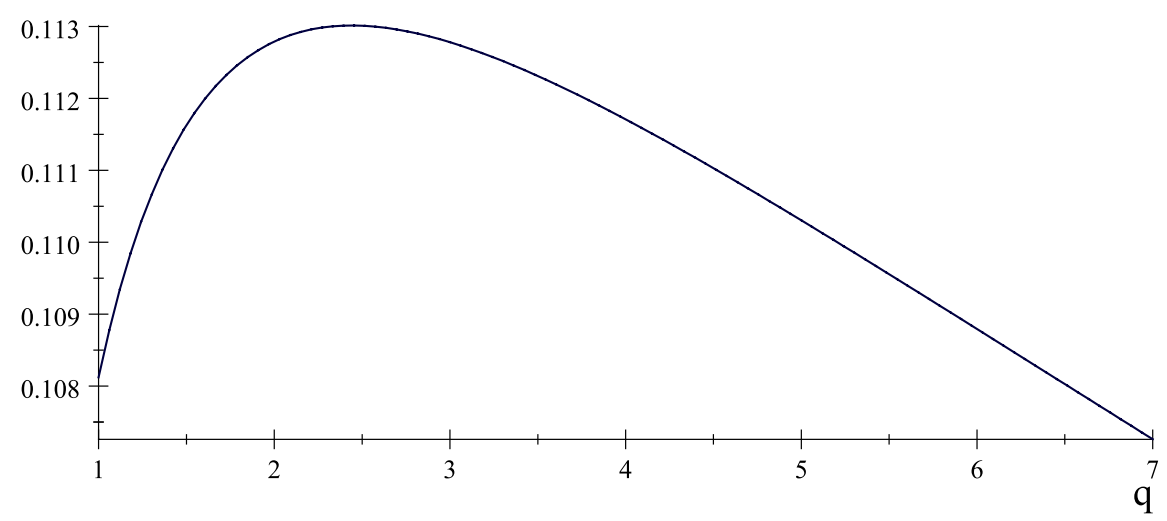

(c)

Figure 1. (a) Active communication by agents 1 and $2\left(r_{13} / r_{23}\right)$. (b) Passive communication by agent $3\left(p_{13} / p_{23}\right)$. (c) Influence of agents 1 and 2 on agent $3\left(b_{13} / b_{23}\right)$. 
1 and 2 talk less and less to the outsider, the outsider begins to invest less in listening. Therefore, the overall pattern of influence is monotonic (Figure $1(\mathrm{c})) .{ }^{15}$

It is also interesting to note that the ratio between active and passive communication is decreasing in $q$ throughout, confirming that the presence of a stronger exogenous tie between agents 1 and 2 causes an increasing imbalance between the desire of outsiders to obtain information and the willingness of agents 1 and 2 to provide it. ${ }^{16}$

\subsection{Underinvestment}

The presence of complementarities among agents is likely to lead to inefficiently low investment in communication. To verify this claim, we must first define the "efficient" benchmark, and there are two candidates. One can compare the equilibrium outcome to the outcome that would arise if communication intensities were chosen by a planner, while decision functions were still delegated to agents. Alternatively, one can use as a benchmark the case where the planner is also responsible for choosing decision functions. Here, we analyze the first case, as we are particularly interested in inefficiencies that derive from underinvestment in communication.

Reconsider our baseline game $\Gamma(\mathbf{D}, \mathbf{k}, \mathbf{s})$. Keep the same payoff functions $u_{i}$ defined in (1), but now assume that each agent $i$ solves

$$
\max _{\left\{b_{i j}^{*}\right\}_{j=1}^{n}} E\left[u_{i}\right]
$$

while a planner solves

$$
\max _{\left\{p_{i j}^{*}\right\}_{i, j},\left\{r_{i j}^{*}\right\}_{i, j}} \sum_{i=1}^{n} E\left[u_{i}\right]
$$

The planner moves first, but—as in the rest of the paper-we assume that agents do not observe communication investments directly. ${ }^{17}$ Call this new game $\Gamma^{*}(\mathbf{D}, \mathbf{k}, \mathbf{s})$.

We can offer an equilibrium characterization that mirrors that of Theorem 1.

Proposition 2. If the planner chooses communication intensities, in equilibrium,

$$
\begin{aligned}
\mathbf{b}_{\cdot j}^{*} & =(\mathbf{I}-\mathbf{\Omega})^{-1} \cdot \mathbf{h}_{\cdot j}^{*} \quad \text { for all } j \\
r_{i j}^{*} & =\sqrt{\sum_{k=1}^{n} d_{i k}+\sum_{k \neq i}^{n} d_{k i}} \frac{b_{i j}^{*}}{k_{r}} \text { for all } i \neq j \\
p_{i j}^{*} & =\sqrt{\sum_{k=1}^{n} d_{i k}+\sum_{k \neq i}^{n} d_{k i}} \frac{b_{i j}^{*}}{k_{p}} \quad \text { for all } i \neq j
\end{aligned}
$$

\footnotetext{
${ }^{15}$ The plot uses the parameters $d=5, s_{1}=s_{2}=s_{3}$, and $k_{p}=k_{r}=0.01$.

${ }^{16}$ Cliques can damage the agents who are excluded from them. Section S3 of the supplementary material presents a three-agent example where a player is better off if communication between the other two players is prohibited.

${ }^{17}$ In another conceivable version of the planner's problem, communication investments could be publicly observable. This will generate an additional discrepancy between the baseline game and the planner's problem.
} 
with

$$
h_{i j}^{*}= \begin{cases}\omega_{j j} & \text { if } i=j \\ -s_{j} \frac{k_{p}+k_{r}}{\sqrt{D_{i}+\sum_{k \neq j}^{n} d_{k j}}} & \text { otherwise. }\end{cases}
$$

Communication creates positive externalities that players do not internalize in the noncooperative game. Comparing Proposition 2 with Theorem 1, we see that there are two channels that generate inefficiency. First, for any given vector of decision coefficients $\mathbf{b}$, communication intensity vectors $\hat{\mathbf{r}}$ and $\hat{\mathbf{p}}$ are larger in the planner's solution, namely, for any given pattern of influence, agents underinvest in communication. Second, when $i \neq j$ the $h_{j i}^{*}$ are smaller in the planner's solution, meaning that there is an additional feedback effect that goes through influence. In the planner's solution, agents expect communication intensities to be higher and, hence, they are more influenced by signals they receive. As both effects go in the same directions, this proves the following proposition.

Proposition 3. In the equilibrium of $\Gamma^{*}(\mathbf{D}, \mathbf{k}, \mathbf{s})$, all the decision coefficients and communication intensities are larger than in the equilibrium of $\Gamma(\mathbf{D}, \mathbf{k}, \mathbf{s})$.

Now that we know that communication investment is too low, we can ask whether underinvestment is more of a problem for active or for passsive communication. Namely, for any directed link between two agents, is the ratio $r_{i j} / p_{i j}$ higher in the baseline case or when the planner chooses communication intensities? From Proposition 2 and Theorem 1, we see the following proposition.

Proposition 4. In the planner's solution the active/passive ratio depends only on relative cost

$$
\frac{r_{i j}}{p_{i j}}=\frac{k_{p}}{k_{r}} .
$$

Instead, in the noncooperative solution, the ratio is

$$
\frac{r_{i j}}{p_{i j}}=\frac{k_{p}}{k_{r}} \sqrt{\frac{d_{j i}}{D_{i}}} .
$$

In general, we should expect $D_{i}>d_{j i} .{ }^{18}$ For instance, if the problem is symmetric $\left(d_{i j}=\bar{d} Q\right.$ for all $i \neq j$ and $\left.d_{i i}=(1-(n-1) \bar{d}) Q\right)$, we have

$$
\gamma_{i j}=\bar{d} \quad \text { for all } i \neq j \text {. }
$$

Consider the case in which active and passive communication are equally costly, i.e., $k_{p}=k_{r}$. As $\bar{d}<1 /(n-1)$, this means that the ratio between active and passive communication is bounded above by $1 /(n-1)$, implying that (i) it is smaller than 1 and (ii) it becomes lower as $n$ increases. The only case where passive communication does not have

\footnotetext{
${ }^{18}$ Only in the case that agent $i$ is particularly prominent and the interaction coefficient $d_{j i}$ for another agent $j$ is larger than the sum of interaction coefficients $D_{i}$ that affect agent $i$, active communication can be relatively more intensive than passive communication.
} 
an intrinsic advantage is when there are only two agents. Conversely, as the number of agents increases, the ratio tends to zero.

As we mentioned after Theorem 1, between active and passive communication, there is strategic asymmetry, which favors passive communication. We will now offer a more precise intuition for this result. Consider two agents, $i$ and $j$, and focus on communication from $j$ to $i$, both $i$ listening $\left(p_{i j}\right)$ and $j$ speaking $\left(r_{i j}\right)$. The payoffs of the two agents, net of communication costs, are given by

$$
\begin{aligned}
& u_{i}=-d_{i i}\left(a_{i}-\theta_{i}\right)^{2}-\sum_{k \neq i} d_{i k}\left(a_{i}-a_{k}\right)^{2} \\
& u_{j}=-d_{j j}\left(a_{j}-\theta_{j}\right)^{2}-\sum_{k \neq j} d_{j k}\left(a_{j}-a_{k}\right)^{2} .
\end{aligned}
$$

The signal that $i$ receives from $j, y_{i j}$, affects $i$ 's action. As our equilibrium is linear,

$$
\frac{d a_{i j}}{d y_{i j}}=b_{i j} .
$$

An increase in the precision of the signal $y_{i j}$ increases the precision of the action $a_{i j}$, which, in turn, is beneficial to our risk-averse agents because it eliminates unnecessary noise in the decision. However, the expressions for $u_{i}$ and $u_{j}$ show that this effect is asymmetric. For the receiver $i$, the effect is quite direct as it involves his action $a_{i}$ directly and it affects all the terms of his payoff:

$$
\frac{d u_{j}}{d y_{i j}}=D_{i} b_{i j} .
$$

Instead, for the sender $j$, the effect is less direct as it does not involve his action $a_{j}$ directly but just the receiver's action:

$$
\frac{d u_{i}}{d y_{i j}}=d_{j i} b_{i j} .
$$

The asymmetry captures the idea that passive communication has a more immediate use than active communication because the receiver can control the action directly, while the sender must reply on the receiver's decision.

Our results imply that organizations - and, in particular, firms - should look for direct and indirect ways to incentivize communication and, in particular, active communication. Indeed, companies often boast about their culture of internal communication. Many management methods introduce protocols that require workers to set aside time to communicating with other agents. A case in point is quality function deployment (QFD), a quality management method that originated in Japan and is now widely used across the world (Akao 1990). There is direct evidence that QFD operates by increasing communication intensity beyond hierarchical lines (Griffin and Hauser 1992). Another example of a structured way to encourage communication is provided by the use of "quality circles" in total quality management (TQM). 
It is interesting to note that management methods such as the above-cited QFD place a great emphasis on giving a voice to all team members, encouraging them to communicate their information independent of their hierarchical position. In our setup, this can be seen as an attempt to reduce the cost of active communication, which we have just seen would be particularly useful to the company, especially if it is large.

Ichniowski et al.'s (1997) influential study of the effect of the adoption of human resources management (HRM) practices finds a positive effect of setting up meetings between managers and workers. Ichniowski and Shaw (2004) consider, in particular, the role of communication and shows that the successful adoption of innovative (and performance-enhancing) HRM practices requires investing in workers' connective capital. The latter, however, comes at a cost: "these investments in connective capital can be costly—involving higher training, screening, and information sharing costs, and potentially higher wages." Thus it appears that moving toward the planner's solution requires the organization to invest in a set of tools and practices that encourage employees to communicate.

The result that underinvestment in active communication is more severe than underinvestment in passive communication depends on our assumption that communication has a pairwise nature. In our setup, there are no economies of scale in sending signals to multiple agents or receiving signals from multiple agents. Phone calls and site visits are good examples of pairwise costs. However, other communication modes display economies of scale on the active side. The cost of sending an e-mail or updating a website is almost independent from the number of recipients or readers. However, such economies of scale are mostly absent on the passive side. As one expects, this would readjust the balance between active and passive communication. ${ }^{19}$ This observation does not invalidate the result that there is a tendency to underinvest in active communication; it just implies that the tendency can be offset by strong economies of scale in active communication.

\section{How influential is AN Agent?}

In this section, we use the characterization in Theorem 1 to measure the overall influence of agents on their peers. Influence has so far only been discussed in bilateral terms: how much does agent $i$ influence agent $j$ ? We now ask how influential an agent is with respect to all other agents. The global influence of agent $i$ will capture the marginal effect of a change in $i$ 's local state on the other agents. The assumption that local states are independent guarantees that influence is purely due to communication and coordination, and not to spurious correlation between actions due to correlation between states.

The global influence of agent $i$, which we denote by $\mathcal{I}_{i}$, is

$$
\mathcal{I}_{i}=\sum_{j=1}^{n} b_{j i}, \quad k=1, \ldots, n .
$$

\footnotetext{
${ }^{19}$ In Section S4 of the supplementary material, we modify the baseline model to allow for economies of scale on the active communication side, and we show that the ratio between active and passive communication is approximately 1 .
} 
In words, the global influence of agent $i$ corresponds to the sum of the expected effects of a change on the agent's local state on all actions (including the agent's own action). The average action of the group conditional on the values of $\vec{\theta}=\left(\theta_{1}, \ldots, \theta_{n}\right)$ is equal to ${ }^{20}$

$$
\mathbb{E}_{\vec{\theta}}\left[\frac{1}{n} \sum_{i} \sum_{j=1}^{n} b_{j i} y_{j i}\right]=\frac{1}{n} \sum_{i} \sum_{j=1}^{n} b_{j i} \theta_{i}=\frac{1}{n} \sum_{i} \mathcal{I}_{i} \theta_{i} .
$$

Our notion of influence is an equilibrium concept. It depends on the communication investment and decision strategy that the players in our game choose. In this section, we will first show that our game-theoretic notion is linked to a widespread axiomatic measure of centrality: eigenvector centrality. We then illustrate, through an example inspired by Dalton (1959), how this correspondence can be used to understand and measure organizational phenomena.

\subsection{Eigenvector centrality}

As mentioned in the Introduction, eigenvector centrality displays properties that are unique among centrality measures (Palacios-Huerta and Volij 2004), and has been applied successfully in search engines and citation indices. To the best of our knowledge, there exists no game-theoretic microfoundation of eigenvector centrality.

Let us now provide a formal definition (in the remarks to Theorem 5, we will discuss how eigenvector centrality relates to other centrality measures). Given a network described by an $n$-square adjacency matrix $G$, let $\tilde{G}$ be the normalized version of the matrix, where all the $g_{i i}$ 's are set to zero and every element $\tilde{g}_{i j}$ is defined as

$$
\tilde{g}_{i j}=\frac{g_{i j}}{\sum_{k \neq i} g_{i k}} .
$$

The eigenvector centrality index is defined as the positive left eigenvector of matrix $\tilde{G}$, namely the smallest strictly positive vector $\iota$ that solves

$$
\iota=\tilde{G}^{\prime} \iota .
$$

Since $\tilde{G}$ is by definition a stochastic matrix, its largest eigenvalue is equal to 1 ; therefore, 1 is also the largest eigenvalue of $\tilde{G}^{\prime}$. Furthermore, the matrix is irreducible as all entries are strictly positive. The Perron-Frobenius theorem ensures then that there exists a unique nonnegative eigenvector of $\tilde{G}^{\prime}$ associated to this eigenvalue. The eigenvector centrality vector is just a normalization of this vector. Note that the vector can always be normalized so that its elements sum to 1 . From now on, we always use this normalization.

One way to understand this definition is to see $\tilde{G}$ as a Markov transition matrix and see $\iota$ as its ergodic state. Imagine an object that bounces probabilistically from one node to the other according to $\tilde{G}$ : the value of $\iota_{i}$ denotes the percentage of time that the object spends, on average, in node $i$.

\footnotetext{
${ }^{20}$ We define $y_{i i}=\theta_{i}$ for all $i$.
} 
Another way to understand eigenvector centrality is to think of the importance of node $i$ as defined by a weighted sum of the other nodes' "importances," where the weights are given by the $g$ 's, namely

$$
\iota_{i}=\sum_{j \neq i} \tilde{g}_{j i} \iota_{j}
$$

In other words, node $i$ "receives" a percentage $\tilde{g}_{j i}$ of the importance of node $j$. If we repeat this exercise for all nodes, we obtain the system (7). Thus, the vector $\iota$ is the fixed point of this importance-distribution procedure.

To connect eigenvector centrality to our game, fix $D, s, k_{r}$, and $k_{p}$, and define the payoff function

$$
u_{i}=-\left(d_{i i}\left(a_{i}-\theta_{i}\right)^{2}+\frac{1}{t} \sum_{j \neq i} d_{i j}\left(a_{i}-a_{j}\right)^{2}+t^{\lambda} k_{r}^{2} \sum_{j \neq i} r_{j i}+t^{\lambda} k_{p}^{2} \sum_{j \neq i} p_{i j}\right),
$$

where $t \in(0, \infty)$ and $\lambda>1$.

For every value of the parameter $t$, we have a well defined instance of our game, which, with a slight abuse of notation, we can call $\Gamma\left(D, s, k_{r}, k_{p}, t\right)$. A decrease in $t$ has two effects: it reduces the relative importance of adaptation over coordination and it reduces the cost of communication (even faster as $\lambda$ is larger than 1 ).

For every $\left(D, s, k_{r}, k_{p}\right)$, we have the following definitions:

- Global influence. For every $t>0$, Theorem 1 provides a unique characterization of the equilibrium in communication and decisions, which, in turn, results in a unique vector of global influences. We let $\mathcal{I}_{i}(t)$ denote the global influence of agent $i$ when the parameter has value $t$.

- Eigenvector centrality. Let $\tilde{G}$ be the matrix with entries $\gamma_{i i}=0$ for all $i$ and let $\gamma_{i j}=d_{i j} / \sum_{k \neq i} d_{i k}$. Based on the discussion above, the eigenvector index of agent $i$ is $\iota_{i}$, defined as the $i$ th component of the vector that solves

$$
\iota=\tilde{G}^{\prime} \iota
$$

and that satisfies $\sum_{j} \iota_{j}=1$. Note that this definition only makes use of the interaction matrix $D$, not of $s, k_{r}$, and $k_{p}$.

The two notions are related by the following theorem.

THeOREm 5. As $t \rightarrow 0$, the relative global influence of agents converges to the ratio of eigenvector centrality indices weighted by an adaptation of $v$ 's coordination ratio. Namely, for any $i$ and $j$,

$$
\lim _{t \rightarrow 0} \frac{\mathcal{I}_{i}(t)}{\mathcal{I}_{j}(t)}=\frac{\iota_{i}}{\iota_{j}} \frac{\frac{d_{i i}}{D_{-i}}}{\frac{d_{j j}}{D_{-j}}}
$$


In particular, if $d_{i i}=d_{j j}$ and $D_{-i}=D_{-j}$ for all $i, j \in N$, then we obtain that

$$
\lim _{t \rightarrow 0} \frac{\mathcal{I}_{i}(t)}{\mathcal{I}_{j}(t)}=\frac{\iota_{i}}{\iota_{j}} .
$$

Theorem 5 builds on two intermediate results. First, we show (Step 1 of the proof; see the Appendix) that the global influence of an agent $\mathcal{I}_{i}(t)$ can be written as a weighted sum of the Bonacich centrality indices of all the agents, where the weight on agent $i$ is positive and the weights on all the other agents are negative. Bonacich centrality is based on counting the number of paths (with appropriate weights) that reach a certain node. ${ }^{21}$ In our case, the weight of a path is the product of all the $d_{i j}$ of the links that form the path. A node is more central if the weighted sum of paths is larger. This first step is inspired by Ballester et al.'s (2006) result on network games with linear-quadratic utilities, but extends the framework to asymmetric information and endogenous communication. Furthermore, we show that when communication costs go down, all the negative terms in the weighted sum of the Bonacich centrality indices vanish and $\mathcal{I}_{i}(t)$ tends to the Bonacich centrality of agent $i$. This is intuitive because when communication becomes costless, our model converges to Ballester et al.'s (2006) complete information setup.

Second, we show that when the relative importance of the adaptation terms $d_{i i}$ goes to zero, the ratio between the Bonacich centralities of two agents tends to the ratio of the eigenvector centralities of those two agents (multiplied by $\left.\left(d_{i i} D_{-j}\right) /\left(D_{-i} d_{j j}\right)\right)$. The underlying idea is that the difference between Bonacich centrality and eigenvector centrality is that the former puts some exogenous baseline centrality on each agent. In our case, it is proportional to their adaptation coefficient $d_{i i}$. As we let the relative importance of adaptation go to zero, Bonacich centrality tends to eigenvector centrality. This intermediate result is closely related to Bonacich (1987), who establishes an asymptotic connection between Bonacich centrality and eigenvector centrality. Bonacich's result is extended by Golub and Lever (2010, Theorem 3), who provide general conditions under which Bonacich centrality converges to eigenvector centrality.

Note that the precision of agent $i$ 's state, $s_{i}$, does not appear in Theorem 5 . This is because $\mathcal{I}_{i}$ measures a marginal effect. Of course, the average influence of an agent on other agents will be greater if the precision $s_{i}$ is lower.

Remark 6 . When $t=0, \mathcal{I}_{i}(t)$ is not well defined. When $t=0$, Theorem 1 does not apply for two reasons. First, if all adaptation terms $d_{i i}$ are zero, the matrix $(\mathbf{I}-\mathbf{\Omega})$ is singular and $b$ 's are not well defined, which captures the fact that agents who care only about coordination can achieve their goals by agreeing to select all the same arbitrary action independent of the realization of local states. Second, if $k_{r}$ and $k_{p}$ are zero, the expressions in (ii) and (iii) of Theorem 1 are not well defined, because the agents would choose infinite precisions.

\footnotetext{
${ }^{21}$ See Jackson (2008, Chapter 2) for a discussion of Bonacich centrality and eigenvector centrality, and for more bibliographical references. Confusingly, Bonacich worked on both measures and his name is associated with both. We follow the convention, adopted by most economists, of calling Bonacich centrality the measure used by Ballester et al. (2006).
} 


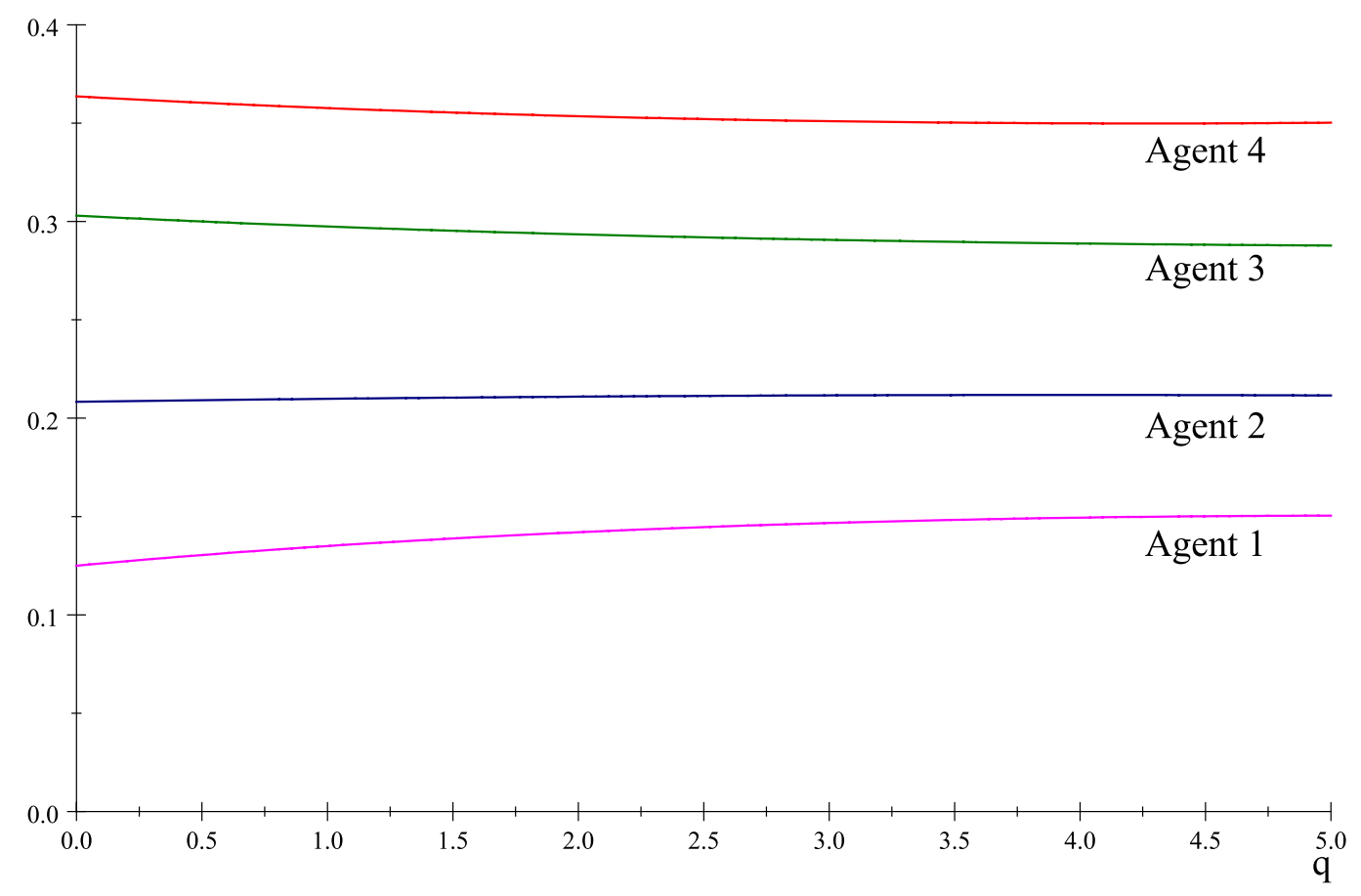

Figure 2. Global influence.

Theorem 5 can be understood as an approximation result. When agents are much more interested in coordinating with other agents than adapting to their own state, relative influence can be approximated by a weighted version of the eigenvector centrality index. To illustrate this point, consider the following numerical example. Suppose that $s_{1}=s_{2}=s_{3}=s_{4}$ and that the interaction matrix is given by

$$
\mathbf{D}=\left[\begin{array}{llll}
q & 1 & 2 & 4 \\
1 & q & 2 & 4 \\
1 & 2 & q & 4 \\
1 & 2 & 4 & q
\end{array}\right]
$$

As this example satisfies the condition that $d_{i i}$ and $D_{-i}$ are constant across agents, we can use the simplified version of Theorem 1. Also suppose that $k_{r}=k_{p}=0.001 q^{2}$. For every positive value of $q$, one can use Theorem 1 to compute the communicationbased global influence of each agent. The relative influence is depicted in Figure 2 as $\mathcal{I}_{i} /\left(\mathcal{I}_{1}+\mathcal{I}_{2}+\mathcal{I}_{3}+\mathcal{I}_{4}\right)$.

The eigenvector centrality indices can be easily computed from $D$ :

$$
\iota_{1}=0.125, \quad \iota_{2}=0.208, \quad \iota_{3}=0.303, \quad \iota_{4}=0.363 .
$$

Figure 2 compares the eigenvector indices and the global influences for positive values of $q$. As Theorem 5 predicts, when $q$ tends to zero, the relative global influences tend to the eigenvector indices. One can also see that the eigenvector centrality index is a 
good approximation even for relatively large values of $q$; in fact, values that are larger than any of the other interaction parameters, like $q>1.22$

\subsection{Influence in hierarchical structure}

Because of its extreme tractability, the eigenvector centrality allows us to obtain simple predictions on which agents will be more influential. Instead of trying to characterize the comparative statics of the whole game, we can focus our attention on the eigenvector centrality indices, which are computed on coordination parameters $\left(d_{i j}\right)_{i \neq j}$ only. Of course, as proven in Theorem 5, these predictions are only approximately correct and the validity of the approximation depends on how strong the coordination motive is vis-á-vis the adaptation motive.

The range of applications is virtually boundless. One natural question, which we can use to illustrate the scope of the theorem, has to do with influence in hierarchies. According to Weber (1978), efficient bureaucracies take the form of hierarchies where influence emanates necessarily from the top. Dalton (1959) famously showed that the Weberian view was at odds with extensive evidence from a U.S. chemical plant. Top managers appeared to be less influential than middle managers. The latter formed cliques with workers and exerted the actual control on production: these arrangements were often motivated by personal goals, but they also helped make the firm run smoothly. While these issues are complex and deserve a more systematic treatment, our setup yields a simple benchmark result on when we should expect middle managers to be more influential than top managers. We first show that hierarchies exhibit an intrinsic bias toward making top agents in higher tiers more influential. We then give an example of what has to happen for this bias to be overcome.

Hierarchical structures can be represented as a subclass of our games. Consider a set of agents grouped into $K$ levels. At level 0 , there is one agent. An agent at level $k$ has $s \geq 2$ subordinates at level $k-1$. Every agent reports to exactly one superior. The agents at level $K$ have no subordinates. If $i$ is a subordinate of $j$, then we assume that $d_{i j}=1$ (a normalization) and $d_{j i}=a .^{23}$ We call the problem we have just defined a regular hierarchy because it looks the same at every level, except the top and the bottom.

\footnotetext{
${ }^{22}$ It is interesting to note that other simple graph-theoretic measures would not perform as well. Take, for instance, one of the simplest measures of the value of a node, the (relative) in-degree, namely the sum of the strength of interactions from other agents toward a particular agent (divided by the total strength of interaction over the graph):

$$
I D_{i}=\frac{\sum_{j} d_{i j}}{\sum_{i, j} d_{i j}} .
$$

The result would be

$$
I D_{1}=0.107 ; \quad I D_{2}=0.178 ; \quad I D_{3}=0.285 ; \quad I D_{4}=0.428,
$$

which is a much worse approximation for all values of $q$ on the range.

${ }^{23}$ For any other two agents, we assume $d_{i j}=0$. For a generic game, the presence of zero interaction coefficients could lead to binding nonnegativity constraints. However, in the asymptotic case under consideration, the cost of communication becomes a negligible fraction of the coordination gains. As we show in the proof of Theorem 5, nonnegativity constraints are not binding in the limit.
} 
Dalton's nonmonotonic influence cannot occur in a regular hierarchy. ${ }^{24}$

Proposition 7. For any $s \geq 2$ and any $2 \leq k \leq K-1$, the ratio between the influence of an agent and his superior is

$$
\frac{I_{k+1}}{I_{k}}=a
$$

In a regular hierarchy, Dalton's anomaly cannot arise: global influence is monotonic in the agent's level-increasing if $a>1$ and decreasing if $a<1$. Given that superiors have many subordinates, but subordinates have only one superior, the natural assumption is that $a<1$. In fact, one might assume that an agent cares as much about his superior as the set of his subordinates: $a s=1$. In that case, the influence ratio is the reciprocal of the span,

$$
I_{k+1}=\frac{1}{s} I_{k}
$$

What is the intuition for this result in terms of our noncooperative communication and influence game? In equilibrium, agents invest in communication mostly along hierarchical lines. Agents will also invest in communicating laterally and skipping levels (e.g., to coordinate with his superior's action, an agent wants to know his superior's state). However, these investments are much lower. Hence, influence too follows hierarchical lines: the local state of a superior affects her subordinates' decisions and the local states of inferiors affect their superior's action. However, one superior affects many subordinates, while one subordinate only affects one superior. This multiplier effect determines monotonicity in influence.

To find a situation à la Dalton where influence is nonmonotonic, we abandon the assumption that the hierarchy is regular. For instance, we can assume that interaction terms are stronger at lower levels than at higher levels. Suppose that $K=3$ and $s=2$. First, consider a regular hierarchy where $d_{i j}=1$ and $d_{j i}=\frac{1}{2}$ for all superior-subordinate pairs. The proposition above is confirmed; the eigenvector centrality indices increase monotonically with the agent's rank,

$$
\begin{aligned}
& I_{0}=I_{1}=\frac{8}{48} \\
& I_{2}=\frac{4}{48} \\
& I_{3}=\frac{1}{48} .
\end{aligned}
$$

\footnotetext{
${ }^{24}$ This ratio does not apply at the top and at the bottom. At the bottom, monotonicity holds a fortiori. At the top, there is a potential problem because the agent at level 0 interacts with only $s$ agents and, hence, he is at a disadvantage. For the top two levels,$$
\frac{I_{1}}{I_{0}}=a+\frac{1}{s}
$$

The assumption that an agent cares equally about his superior and the set of his subordinates (as $=1$ ) guarantees monotonicity at the top.
} 
Now modify the hierarchy above by assuming that if $i$ belongs to $k=3$ and $j$ belongs to $k=2, d_{i j}=4$ and $d_{j i}=2$. For this irregular hierarchy, the eigenvector centrality yields

$$
\begin{aligned}
& I_{0}=I_{1}=\frac{4}{48} \\
& I_{2}=\frac{5}{48} \\
& I_{3}=\frac{2}{48} .
\end{aligned}
$$

Hence, now agents at tier 2 are more influential than agents in the top two tiers.

What breaks the Weberian influence monotonicity in this example is that the two bottom levels have strong ties to each other. They invest a lot in communicating with each other and they influence each other. These strong ties do not extend to the top two levels and agents at the two bottom level are not very influenced by the local states of top managers. In Dalton's language, each level-2 manager with his two level-3 workers constitutes a vertical symbiotic clique, i.e., a two-way tie between an officer and his subordinates that represents the "most common and enduring clique in large structures" (Dalton 1959, p. 59).

The results in this section-both the proposition and the counterexample-are approximations that were obtained by looking only at coordination coefficients of the form $d_{i j}$, with $i \neq j$. The analysis was much simpler than if we had tried to operate directly on the equilibrium of the noncooperative game. But we also know that the approximations are valid, at least locally. For values of the adaptation coefficients $d_{i i}$ and the communication parameters $k$ that are sufficiently low, Theorem 1 guarantees that the relations that we have uncovered for eigenvector centrality indices are reflected in similar relations for global influence.

\section{Conclusion}

The present paper is a step toward modeling equilibrium endogenous costly information flows among multiple agents, but much work remains to be done. Of course, it would be interesting to know what happens beyond the normal-quadratic setup and to show under what conditions the normal-quadratic is a good approximation of other settings. Other communication structures are explored in the supplementary material (broadcasting and alternative communication protocols). However, it would be interesting to take a more general approach and perform comparative statics on communication modes, for instance, asking how communication and influence change when the communication technology changes.

Does our model have the potential to be used for empirical work? What kind of data could identify the model? Suppose that we observe the information flows among nodes of a network (e.g., what amount of resources each firm spends for liaising with other firms) but not the underlying interaction matrix, communication cost parameters, local state uncertainty, or decision functions. The potential for identification is there. If the 
number of agents is at least four, Theorem 1 supplies a number of restrictions that is at least as large as the number of primitive variables to be estimated. ${ }^{25}$

A similar identification potential exists in the other formulation of the problem, which is explored in the Appendix. This observation, although preliminary, appears to indicate that data on information flows could be a fruitful avenue for investigating organizations empirically if combined with a model—not necessarily the present one-of endogenous communication in network games (Garicano and Prat 2013).

In our paper, asymmetric information flows occur as a response to asymmetric interaction structures. However, Dessein et al. (2013) show that with different communication cost structures, asymmetric information flows arise in symmetric situations, thus forming the basis for a rational inattention theory of endogenous leadership.

Finally, we have considered a static setup, and although this seems to be a natural starting point for our inquiry, it would be interesting to analyze dynamic communication protocols in a similar environment. Information would then come from direct communication and from learning of the past activity of some or all agents in the organization. This relates to a recent literature on social learning in networks (see, for example, Bala and Goyal 1998 and Acemoglu et al. 2011) and we plan to pursue this analysis in future research.

\section{Appendix: Proofs \\ Proof of Theorem 1}

Agent $i$ 's payoff gross of communication costs is given by

$$
u_{i}=-\left(d_{i i}\left(a_{i}-\theta_{i}\right)^{2}+\sum_{j \neq i} d_{i j}\left(a_{i}-a_{j}\right)^{2}\right) .
$$

As is well known for quadratic games, the action that maximizes $i$ 's expected payoff is

$$
a_{i}=d_{i i} \theta_{i}+\sum_{j \neq i} d_{i j} E\left[a_{j}\right]
$$

In the conjectured equilibrium, in the second stage of the game, agent $i$ knows that he has chosen $\left(\tilde{r}_{j i}\right)_{j \neq i}$ and $\left(\tilde{p}_{i j}\right)_{j \neq i}$ (which may be different from the equilibrium values). He assumes that the other agents have chosen communication intensities according to the equilibrium values and that they will choose actions according to the equilibrium linear strategies. The optimal action is then

$$
\begin{aligned}
a_{i} & =d_{i i} \theta_{i}+\sum_{j \neq i} d_{i j} E\left[b_{j j} \theta_{j}+\sum_{k \neq j} b_{j k} y_{j k}\right] \\
& =d_{i i} \theta_{i}+\sum_{j \neq i} d_{i j} E\left[b_{j j} \theta_{j}+\sum_{k \neq j} b_{j k}\left(\theta_{k}+\varepsilon_{j k}+\eta_{j k}\right)\right] .
\end{aligned}
$$

\footnotetext{
${ }^{25}$ Palacios-Huerta and Prat (2010) exploit e-mail traffic data within a company to compute the eigenvector centrality of individual workers, and show that it predicts the workers' success within the company as measured in terms of rank, income, and career path.
} 
The solution to this classical signal extraction problem is a linear function

$$
a_{i}=\tilde{b}_{i i} \theta_{i}+\sum_{j \neq i} \tilde{b}_{i j} y_{i j}
$$

where coefficients are defined by

$$
\begin{aligned}
D_{i} \tilde{b}_{i i} & =d_{i i}+\sum_{j \neq i} d_{i j} b_{j i} \\
D_{i} \tilde{b}_{i j} & =\frac{r_{i j} \tilde{p}_{i j}}{s_{j} r_{i j}+s_{j} \tilde{p}_{i j}+r_{i j} \tilde{p}_{i j}} \sum_{k \neq i} d_{i k} b_{k j} \quad \text { for all } j \neq i .
\end{aligned}
$$

Now consider the first stage of the game. While precisions yield more compact final expressions, the first part of this proof is more readable if we use variances. We denote by $\sigma_{i}=1 / s_{i}$ the variance of $\theta_{i}$, by $\rho_{i j}=1 / r_{i j}$ the variance of $\varepsilon_{i j}$, and by $\pi_{i j}=1 / p_{i j}$ the variance of $\eta_{i j}$ (the omission of the square sign is intentional: $\sigma, \rho$, and $\pi$ are variances, not standard deviations). The payoff for agent $i$ in $\Gamma(\mathbf{D}, \mathbf{k}, \mathbf{s})$ is given by

$$
u_{i}=-\left(d_{i i}\left(a_{i}-\theta_{i}\right)^{2}+\sum_{j \neq i} d_{i j}\left(a_{i}-a_{j}\right)^{2}+\sum_{j \neq i} k_{r}^{2}\left(\frac{1}{\rho_{j i}}\right)+\sum_{j \neq i} k_{p}^{2}\left(\frac{1}{\pi_{i j}}\right)\right) .
$$

Given the strategies to be used in the second stage,

$$
\begin{aligned}
-u_{i}= & d_{i i}\left(\left(\tilde{b}_{i i}-1\right) \theta_{i}+\sum_{k \neq i} \tilde{b}_{i k}\left(\theta_{k}+\varepsilon_{i k}+\eta_{i k}\right)\right)^{2} \\
& +\sum_{j \neq i} d_{i j}\left(\sum_{k}\left(\tilde{b}_{i k}-b_{j k}\right) \theta_{k}+\sum_{k \neq i} \tilde{b}_{i k} \varepsilon_{i k}+\sum_{k \neq i} \tilde{b}_{i k} \eta_{i k}-\sum_{k \neq j} b_{j k} \varepsilon_{j k}-\sum_{k \neq j} b_{j k} \eta_{j k}\right)^{2} \\
& +\sum_{j \neq i} k_{r}^{2}\left(\frac{1}{\rho_{j i}}\right)+\sum_{j \neq i} k_{p}^{2}\left(\frac{1}{\pi_{i j}}\right) .
\end{aligned}
$$

Given the assumption that noise terms are stochastically independent, the expected payoff of $i$ is

$$
\begin{aligned}
-E\left[u_{i}\right]= & d_{i i}\left(\left(\tilde{b}_{i i}-1\right)^{2} \sigma_{i}+\sum_{k \neq i}^{2} \tilde{b}_{i k}^{2}\left(\sigma_{k}+\rho_{i k}+\pi_{i k}\right)\right) \\
& +\sum_{j \neq i} d_{i j}\left(\sum_{k}\left(\tilde{b}_{i k}-b_{j k}\right)^{2} \sigma_{k}+\sum_{k \neq i} \tilde{b}_{i k}^{2}\left(\rho_{i k}+\pi_{i k}\right)+\sum_{k \neq j} b_{j k}^{2}\left(\rho_{j k}+\pi_{j k}\right)\right) \\
& +k_{r}^{2} \sum_{j \neq i} \frac{1}{\rho_{j i}}+k_{p}^{2} \sum_{j \neq i} \frac{1}{\pi_{i j}} .
\end{aligned}
$$


By the envelope theorem, we can disregard effects of the form $\left(d \tilde{b}_{i k}\right) /\left(d \rho_{i k}\right)$. The firstorder conditions are

$$
\begin{gathered}
-\frac{\partial E\left[u_{i}\right]}{\partial \rho_{j i}}=d_{i j} b_{j i}^{2}+k_{r}^{2}\left(\frac{1}{\rho_{j i}}\right)^{2}=0 \\
-\frac{\partial E\left[u_{i}\right]}{\partial \pi_{i j}}=D_{i} \tilde{b}_{i j}^{2}+k_{p}^{2}\left(\frac{1}{\pi_{i j}}\right)^{2}=0 .
\end{gathered}
$$

Now replace precisions in (11) and (12), and combine them with (8) and (9) for all agents to obtain a system of equations that characterize the equilibrium:

$$
\begin{aligned}
D_{i} b_{i i} & =d_{i i}+\sum_{j \neq i} d_{i j} b_{j i} \quad \text { for all } i \\
D_{i} b_{i j} & =\frac{r_{i j} p_{i j}}{s_{j} r_{i j}+s_{j} p_{i j}+r_{i j} p_{i j}} \sum_{k \neq i} d_{i k} b_{k j} \text { for all } i, j \neq i \\
\frac{\sqrt{d_{j i}} b_{i j}}{k_{r}} & =r_{i j} \quad \text { for all } i, j \neq i \\
\frac{\sqrt{D_{i}} b_{i j}}{k_{p}} & =p_{i j} \quad \text { for all } i, j \neq i .
\end{aligned}
$$

Restrict attention to a particular $i$. Plugging (15) and (16) into (14), we obtain

$$
\left(s_{j} \frac{k_{p}}{\sqrt{D_{i}}}+s_{j} \frac{k_{r}}{\sqrt{d_{j i}}}+b_{i j}\right) D_{i}=\sum_{k \neq i} d_{i k} b_{k j} \quad \text { for all } j \neq i
$$

which can be rewritten as

$$
b_{i j}-\sum_{k \neq i} \frac{d_{i k}}{D_{i}} b_{k j}=-s_{j}\left(\frac{k_{p}}{\sqrt{D_{i}}}+\frac{k_{r}}{\sqrt{d_{j i}}}\right) \text { for all } j \neq i
$$

Also note that (13) can be rewritten as

$$
b_{i i}-\sum_{k \neq i} \frac{d_{i k}}{D_{i}} b_{k i}=\frac{d_{i i}}{D_{i}}
$$

Using the definitions of $\omega_{i j}$ and $h_{i j}$, the system composed of (17) and (18) becomes

$$
\begin{aligned}
& b_{i i}-\sum_{k \neq i} \omega_{i k} b_{k i}=h_{i i} \\
& b_{i j}-\sum_{k \neq i} \omega_{i k} b_{k j}=h_{i j} \quad \text { for all } j \neq i
\end{aligned}
$$

which in matrix notation corresponds to

$$
(\mathbf{I}-\mathbf{\Omega}) \mathbf{b}_{\cdot j}=\mathbf{h}_{\cdot j},
$$


with solution

$$
\mathbf{b}_{\cdot j}=(\mathbf{I}-\mathbf{\Omega})^{-1} \cdot \mathbf{h}_{\cdot j} .
$$

The last expression, combined with (15) and (16), constitutes the statement of the theorem.

\section{Proof of Proposition 2}

The agents' best responses in the second round, given communication intensities and other agents' strategies are the same as in the baseline case, namely (13) and (14). Instead, of course, the first-order condition for communication intensities given the $b$ 's has changed. The planner solves

$$
\begin{aligned}
& -\frac{\partial}{\partial \rho_{j i}} \sum_{k=1}^{n} E\left[u_{k}\right]=\left(\sum_{k=1}^{n} d_{i k}+\sum_{k \neq i}^{n} d_{k i}\right)\left(b_{j i}^{*}\right)^{2}-k_{r}\left(\frac{1}{\rho_{j i}}\right)^{2}=0 \\
& -\frac{\partial}{\partial \pi_{i j}} \sum_{k=1}^{n} E\left[u_{k}\right]=\left(\sum_{k=1}^{n} d_{i k}+\sum_{k \neq i}^{n} d_{k i}\right)\left(b_{i j}^{*}\right)^{2}-k_{p}\left(\frac{1}{\pi_{i j}}\right)^{2}=0,
\end{aligned}
$$

which can be rewritten as

$$
\begin{aligned}
& r_{i j}^{*}=\sqrt{\sum_{k=1}^{n} d_{i k}+\sum_{k \neq i}^{n} d_{k i}} \frac{b_{i j}^{*}}{k_{r}} \quad \text { for all } i \neq j \\
& p_{i j}^{*}=\sqrt{\sum_{k=1}^{n} d_{i k}+\sum_{k \neq i}^{n} d_{k i}} \frac{b_{i j}^{*}}{k_{p}} \quad \text { for all } i \neq j .
\end{aligned}
$$

The values of $h^{*}$ are obtained by replacing $p^{*}$ and $r^{*}$ in (13) and (14).

\section{Proof of Proposition 3}

We have that $h_{j i}^{*}>h_{j i}$ for all $j \neq i$ and that $h_{i i}^{*}=h_{i i}$. This immediately implies that $b_{j i}^{*}>$ $b_{j i}$ for all $i, j$, and, therefore, that $r_{i j}^{*}>r_{i j}$ and $p_{i j}^{*}>p_{i j}$ for all $i, j$ because $\sum_{k=1}^{n} d_{i k}+$ $\sum_{k \neq i}^{n} d_{k i}>d_{j i}$ and $\sum_{k=1}^{n} d_{i k}+\sum_{k \neq i}^{n} d_{k i}>D_{i}$.

\section{Proof of Theorem 5}

The proof consists of five steps.

Step 1. Bonacich centrality, developed in sociology (Bonacich 1987) and applied to economics by Ballester et al. (2006), is defined as follows. Consider a network described by an $n$-square adjacency matrix $G$, where $g_{i j} \in[0,1]$ measures the strength of the path from $i$ to $j$. Define the matrix

$$
\mathbf{M}(\mathbf{G})=[\mathbf{I}-\mathbf{G}]^{-1} .
$$


Each element $m_{i j}$ of the matrix $\mathbf{M}$ can be interpreted as a sum of the paths-direct or indirect-leading from node $i$ to node $j$. Let $m_{i j}(\mathbf{G})$ be the $i j$ element of $\mathbf{M}$.

The Bonacich centrality measure of node $i$ is defined as

$$
\beta_{i}(\mathbf{G})=\sum_{j=1}^{n} m_{i j}(\mathbf{G})
$$

The centrality of node $i$ is determined by the weighted sum of paths to all nodes that begin in $i$.

Global influence and Bonacich centrality are connected by the following result. ${ }^{26}$

Lемма 8. The global influence of agent $i$ can be expressed as a weighted sum of all the agents' Bonacich centrality measures, computed on $\mathbf{\Omega}^{\prime}$ with decay factor 1 , where the weights are given by $h_{. i}$ :

$$
\mathcal{I}_{i}=\sum_{j=1}^{n} \beta_{j}\left(\mathbf{\Omega}^{\prime}\right) h_{j i}
$$

The influence of agent $i$ is a sum of weighted Bonacich measures, where the weight on the agent's own measure is positive (because $h_{i i}=\omega_{i i}$ ) while all the other weights are negative. Hence, an agent's global influence depends positively on the centrality of that agent and negatively on the centrality of all other agents.

Proof of Lemma 8. From Theorem 1, we know that for all $i$,

$$
\mathbf{b}_{i}=(\mathbf{I}-\mathbf{\Omega})^{-1} \cdot \mathbf{h}_{i} .
$$

We can write

$$
\begin{aligned}
{\left[\begin{array}{c}
b_{1 i} \\
\vdots \\
b_{n i}
\end{array}\right]=} & {\left[\begin{array}{ccc}
\left((\mathbf{I}-\mathbf{\Omega})^{-1}\right)_{11} & \cdots & \left((\mathbf{I}-\mathbf{\Omega})^{-1}\right)_{1 n} \\
\vdots & & \vdots \\
\left((\mathbf{I}-\mathbf{\Omega})^{-1}\right)_{n 1} & \cdots & \left((\mathbf{I}-\mathbf{\Omega})^{-1}\right)_{n n}
\end{array}\right] \cdot\left[\begin{array}{c}
h_{1 i} \\
\vdots \\
h_{n i}
\end{array}\right] } \\
= & \sum_{j=1}^{n} h_{j i}\left[\begin{array}{c}
\left((\mathbf{I}-\mathbf{\Omega})^{-1}\right)_{1 j} \\
\vdots \\
\left((\mathbf{I}-\mathbf{\Omega})^{-1}\right)_{n j}
\end{array}\right]=\sum_{j=1}^{n} h_{j i}\left[\begin{array}{c}
\left((\mathbf{I}-\mathbf{\Omega})^{-1}\right)_{j 1}^{\prime} \\
\vdots \\
\left((\mathbf{I}-\mathbf{\Omega})^{-1}\right)_{j n}^{\prime}
\end{array}\right]
\end{aligned}
$$

so that

$$
\mathcal{I}_{i}=\sum_{k=1}^{n} b_{k i}=\sum_{k=1}^{n} \sum_{j=1}^{n} h_{j i}\left((I-\mathbf{\Omega})^{-1}\right)_{j k}^{\prime}=\sum_{j=1}^{n}\left(\sum_{k=1}^{n}\left((\mathbf{I}-\mathbf{\Omega})^{-1}\right)_{j k}^{\prime}\right) h_{j i} .
$$

\footnotetext{
${ }^{26}$ If we compare Lemma 8 to Ballester et al.'s (2006) Theorem 1, there are three differences. First, their result is based on symmetric information, while ours is based on asymmetric information and endogenous communication. Second, their measure is a Bonacich index, while ours is a weighted sum of Bonacich indices. Third, their decay rate varies according to the model, while ours is always 1 . For all these reasons, it is not easy to establish an intuitive connection between the two results.
} 
If we define the $\mathbf{G}$ matrix in the Bonacich measure to be the transpose of the $\mathbf{\Omega}$ matrix used in Theorem 1 and we let $a=1$, we have

$$
\mathbf{M}(\mathbf{G}, a)=\mathbf{M}\left(\mathbf{\Omega}^{\prime}, 1\right)=\left[\mathbf{I}-\mathbf{\Omega}^{\prime}\right]^{-1}=\left([\mathbf{I}-\mathbf{\Omega}]^{-1}\right)^{\prime}
$$

and, hence,

$$
\sum_{k=1}^{n}\left((\mathbf{I}-\mathbf{\Omega})^{-1}\right)_{j k}^{\prime}=\beta_{j}\left(\mathbf{\Omega}^{\prime}, 1\right)
$$

so that

$$
\mathcal{I}_{i}=\sum_{j=1}^{n} \beta_{j}\left(\mathbf{\Omega}^{\prime}, 1\right) h_{j i} .
$$

Step 2. For any given $t$, the matrix $\tilde{\mathbf{\Omega}}(t)$ is equal to

$$
\tilde{\boldsymbol{\Omega}}(t)=\left[\begin{array}{cccc}
0 & \frac{d_{12}}{t d_{11}^{0}+\sum_{j \neq 1} d_{1 j}} & \cdots & \frac{d_{1 n}}{t d_{11}^{0}+\sum_{j \neq 2} d_{1 j}} \\
\frac{d_{21}}{t d_{22}^{0}+\sum_{j \neq 2} d_{2 j}} & 0 & \cdots & \frac{d_{2 n}}{t d_{22}^{0}+\sum_{j \neq 2} d_{2 j}} \\
\vdots & \vdots & \ddots & \vdots \\
\frac{d_{n 1}}{t d_{n n}^{0}+\sum_{j \neq n} d_{n j}} & \frac{d_{n 2}}{t d_{n n}^{0}+\sum_{j \neq n} d_{n j}} & \cdots & 0
\end{array}\right] .
$$

Note that $\tilde{\mathbf{\Omega}}(0)=\tilde{G}$ (as defined in the text).

For any $t>0$, the Bonacich centrality vector associated to $\tilde{\boldsymbol{\Omega}}(t)$, which we denote $\boldsymbol{\beta}(\tilde{\mathbf{\Omega}}(t))$, is the solution to the system

$$
\left(\mathbf{I}-\tilde{\boldsymbol{\Omega}}^{\prime}(t)\right) \boldsymbol{\beta}(\tilde{\mathbf{\Omega}}(t))=\mathbf{1} .
$$

Note that the Bonacich centrality measure is well defined if and only if the determinant of $\left(\mathbf{I}-\tilde{\mathbf{\Omega}}^{\prime}(t)\right)^{-1}$ is different than 0 , and in such a case, the solution to the previous system is

$$
\boldsymbol{\beta}(\tilde{\mathbf{\Omega}}(t))=\left(\mathbf{I}-\tilde{\mathbf{\Omega}}^{\prime}(t)\right)^{-1} \mathbf{1} .
$$

The eigenvector centrality influence vector is related to the system

$$
\left(\mathbf{I}-\tilde{\mathbf{\Omega}}^{\prime}(0)\right) \mathbf{x}=\mathbf{0} .
$$

Let $E$ be the set of solutions to this system. Since the largest eigenvalue of $\tilde{\mathbf{\Omega}}^{\prime}(0)$ is equal to 1 , the set $E$ is equal to the eigenspace of this largest eigenvalue. The eigenvector centrality influence vector, which we denote $\iota$, is the only element in this eigenspace that satisfies the additional normalization condition $\sum_{i=1}^{n} \iota_{i}=1$.

We are going to prove the following result.

Lemma 9. The limit of $\boldsymbol{t} \boldsymbol{\boldsymbol { \beta }}(\tilde{\mathbf{\Omega}}(t))$ as $t$ tends to 0 is a strictly positive vector, i.e., all its entries are nonnegative and at least one of them is strictly positive. 
Proof. Let $D_{-i}=\sum_{j \neq i} d_{i j}$ and $D_{i}(t)=t d_{i i}^{0}+D_{-i}$. Define $\hat{\mathbf{\Omega}}(t)$ as the matrix with entries $\hat{\omega}_{i j}(t)=d_{i j} / D_{i}(t)$ for all $i, j$. (The difference with respect to $\tilde{\mathbf{\Omega}}(t)$ is that it includes the elements $t d_{i i}^{0} / D_{i}(t)$ in the diagonal, which are zeros in $\tilde{\boldsymbol{\Omega}}(t)$.) Note that by definition, $\hat{\mathbf{\Omega}}(t)$ is a stochastic matrix for all $t$. Also define $\operatorname{Diag}(t)$ as the diagonal matrix $\operatorname{diag}\left(D_{1}(t) / d_{11}^{0}, \ldots, D_{n}(t) / d_{n n}^{0}\right)$. It immediately follows that

$$
\tilde{\mathbf{\Omega}}(t)=\hat{\mathbf{\Omega}}(t)-t \mathbf{D i a g}^{-1}(t) .
$$

Hence, we obtain that

$$
\begin{aligned}
\mathbf{I}-\tilde{\mathbf{\Omega}}(t) & =\mathbf{I}-\hat{\mathbf{\Omega}}(t)+t \mathbf{D i a g}^{-1}(t) \\
& =\left(\mathbf{I}+t \mathbf{D i a g}^{-1}(t)\right) \mathbf{I}-\hat{\mathbf{\Omega}}(t) \\
& =\left(\mathbf{I}+t \mathbf{D i a g}^{-1}(t)\right) \cdot\left(\mathbf{I}-\left(\mathbf{I}+t \mathbf{D i a g}^{-1}(t)\right)^{-1} \hat{\mathbf{\Omega}}(t)\right) .
\end{aligned}
$$

Therefore, we obtain that

$$
(\mathbf{I}-\tilde{\mathbf{\Omega}}(t))^{-1}=\left(\mathbf{I}-\left(\mathbf{I}+t \mathbf{D i a g}^{-1}(t)\right)^{-1} \hat{\mathbf{\Omega}}(t)\right)^{-1} \cdot\left(\mathbf{I}+t \mathbf{D i a g}^{-1}(t)\right)^{-1} .
$$

The matrix $\left(\mathbf{I}+t \mathbf{D i a g}^{-1}(t)\right)^{-1}$ is again a diagonal matrix, with entries equal to

$$
\left(\mathbf{I}+t \mathbf{D i a g}^{-1}(t)\right)_{i i}^{-1}=\frac{1}{1+\frac{t d_{i i}^{0}}{D_{i}(t)}}=\frac{t d_{i i}^{0}+D_{-i}}{2 t d_{i i}^{0}+D_{-i}} .
$$

Each of these diagonal entries tends to 1 when $t$ tends to 0 .

The matrix $\left(\mathbf{I}+t \mathbf{D i a g}^{-1}(t)\right)^{-1} \hat{\mathbf{\Omega}}$ is bounded from below (entry by entry) by $\left(\min _{i}\left(t d_{i i}^{0}+D_{-i}\right) /\left(2 t d_{i i}^{0}+D_{-i}\right)\right) \hat{\boldsymbol{\Omega}}$ and it is bounded from above by $\left(\max _{i}\left(t d_{i i}^{0}+D_{-i}\right) /\right.$ $\left.\left(2 t d_{i i}^{0}+D_{-i}\right)\right) \hat{\mathbf{\Omega}}$. Since all these matrices are nonnegative, this implies that

$$
\left(\mathbf{I}-\left(\mathbf{I}+t \operatorname{Diag}^{-1}(t)\right)^{-1} \hat{\mathbf{\Omega}}\right)^{-1}=\sum_{k \geq 0}\left[\left(\mathbf{I}+t \operatorname{Diag}^{-1}(t)\right)^{-1} \hat{\mathbf{\Omega}}(t)\right]^{k}
$$

is bounded from below by

$$
\sum_{k \geq 0}\left(\min _{i} \frac{t d_{i i}^{0}+D_{-i}}{2 t d_{i i}^{0}+D_{-i}}\right)^{k}(\hat{\boldsymbol{\Omega}}(t))^{k}
$$

and bounded from above by

$$
\sum_{k \geq 0}\left(\max _{i} \frac{t d_{i i}^{0}+D_{-i}}{2 t d_{i i}^{0}+D_{-i}}\right)^{k}(\hat{\mathbf{\Omega}}(t))^{k} .
$$

Remember that we want to show that the limit of $t\left(\mathbf{I}-\mathbf{\Omega}^{\prime}(t)\right)^{-1} \mathbf{1}$ as $t$ tends to 0 is a strictly positive vector (meaning that all its entries are nonnegative and at least one entry is strictly positive). An alternative expression of this vector is

$$
t\left(\mathbf{I}+t \operatorname{Diag}^{-1}(t)\right)^{-1} \cdot\left(\mathbf{I}-\hat{\mathbf{\Omega}}^{\prime}(t)\left(\mathbf{I}+t \operatorname{Diag}^{-1}(t)\right)^{-1}\right)^{-1} \mathbf{1} .
$$


We have proved before that $\left(\mathbf{I}+t \mathbf{D i a g}^{-1}(t)\right)^{-1}$ tends to the identity matrix, so we have to focus on the limit of

$$
t \cdot\left(\mathbf{I}-\hat{\mathbf{\Omega}}^{\prime}(t)\left(\mathbf{I}+t \mathbf{D i a g}^{-1}(t)\right)^{-1}\right)^{-1} \mathbf{1}
$$

We have

$$
\begin{aligned}
t\left(\sum_{k \geq 0}\left(\min _{i} \frac{t d_{i i}^{0}+D_{-i}}{2 t d_{i i}^{0}+D_{-i}}\right)^{k} \hat{\mathbf{\Omega}}^{k}\right) \mathbf{1} & \leq t \cdot\left(\mathbf{I}-\left(\mathbf{I}+t \mathbf{D i a g}^{-1}(t)\right)^{-1} \hat{\mathbf{\Omega}}\right)^{-1} \mathbf{1} \\
& \leq t\left(\sum_{k \geq 0}\left(\max _{i} \frac{t d_{i i}^{0}+D_{-i}}{2 t d_{i i}^{0}+D_{-i}}\right)^{k} \hat{\mathbf{\Omega}}^{k}\right) \mathbf{1} .
\end{aligned}
$$

Since $\hat{\mathbf{\Omega}}$ is stochastic, we know that $\hat{\boldsymbol{\Omega}}^{k} \mathbf{1}=\mathbf{1}$ for all $k \geq 0$. Therefore,

$$
t\left(\frac{1}{1-\frac{t d_{\min }^{0}+D_{\min }}{2 t d_{\min }^{0}+D_{\min }}}\right) \mathbf{1} \leq t \cdot\left(\mathbf{I}-\left(\mathbf{I}+t \mathbf{D i a g}^{-1}(t)\right)^{-1} \hat{\mathbf{\Omega}}\right)^{-1} \mathbf{1} \leq t\left(\frac{1}{1-\frac{t d_{\max }^{0}+D_{\max }}{2 t d_{\max }^{0}+D_{\max }}}\right) \mathbf{1},
$$

where we define $D_{\min }$ and $d_{\min }^{0}$ (resp. max) as the values of $D_{-i^{*}}$ and $d_{i^{*} i^{*}}^{0}$ for the $i^{*}$ that minimizes (resp. maximizes) $\left(t d_{i i}^{0}+D_{-i}\right) /\left(2 t d_{i i}^{0}+D_{-i}\right)$. If we premultiply all vectors by $\mathbf{1}^{\prime}$ and take limits, we get that

$$
n \frac{D_{\min }}{d_{\min }^{0}} \leq \lim _{t \rightarrow 0} t \mathbf{1}^{\prime} \cdot\left(\mathbf{I}-\left(\mathbf{I}+t \mathbf{D i a g}^{-1}(t)\right)^{-1} \hat{\mathbf{\Omega}}\right)^{-1} \cdot \mathbf{1} \leq n \frac{D_{\max }}{d_{\max }^{0}} .
$$

Given a square matrix $\mathbf{X}=\left(x_{i j}\right)$, we have that $\mathbf{1}^{\prime} \cdot \mathbf{X} \cdot \mathbf{1}=\sum_{i, j} x_{i j}=\sum_{i, j} x_{i j}=\mathbf{1}^{\prime} \cdot \mathbf{X}^{\prime} \cdot \mathbf{1}$. Therefore, the argument above shows that the limit of $t \mathbf{1}^{\prime} \cdot\left(\mathbf{I}-\hat{\mathbf{\Omega}}^{\prime}\left(\mathbf{I}+t \operatorname{Diag}^{-1}(t)\right)^{-1}\right)^{-1}$. $\mathbf{1}$ as $t$ tends to 0 is also bounded from below by $n\left(D_{\min } / d_{\min }^{0}\right)$ and from above by $n\left(D_{\max } / d_{\max }^{0}\right)$. This means that the limit of $t\left(\mathbf{I}-\hat{\mathbf{\Omega}}^{\prime}\left(\mathbf{I}+t \mathbf{D i a g}^{-1}(t)\right)^{-1}\right)^{-1} \cdot \mathbf{1}$ is a strictly positive matrix and, therefore, the limit of $t \cdot \hat{\mathbf{\Omega}}^{\prime}\left(\mathbf{I}-\left(\mathbf{I}+t \mathbf{D i a g}^{-1}(t)\right)^{-1}\right)^{-1} \cdot \mathbf{1}$ is a strictly positive vector, as we wanted to show. (We are assuming here that $D_{\min }$ is strictly positive; otherwise, the equilibrium of the game would entail no communication.)

Step 3.

Lemma 10. The limit of $\boldsymbol{\beta} \boldsymbol{\beta}(t)$ as $t$ tends to 0 is proportional to the eigenvector centrality index.

Proof. Now define

$$
S_{0}=\{t \in \mathbb{R} \text { s.t. } \operatorname{det}(\mathbf{I}-\tilde{\mathbf{\Omega}}(t))=0\} .
$$

Note that $0 \in S_{0}$.

We know from the previous lemma that $\lim _{t \rightarrow 0} t \boldsymbol{\beta}(t)$ exists and is a well defined strictly positive vector. By definition, $t \boldsymbol{\beta}(t)$ satisfies

$$
\left(\mathbf{I}-\tilde{\boldsymbol{\Omega}}^{\prime}(t)\right) t \boldsymbol{\beta}(t)=t \mathbf{1}
$$


for all $t \in \mathbb{R} \backslash S_{0}$. We can take limits at both sides of this equation to obtain

$$
\left(\mathbf{I}-\tilde{\boldsymbol{\Omega}}^{\prime}(0)\right)\left(\lim _{t \rightarrow 0} \boldsymbol{\beta}(t)\right)=\mathbf{0} .
$$

This means that the limit vector $\lim _{t} \hat{\boldsymbol{\beta}}(t)$ satisfies the condition that characterizes the set $E$ and, therefore, is proportional to the eigenvector centrality vector.

Step 4. We are now ready to prove the statement of the theorem. From Lemma 8, we know that

$$
\mathcal{I}_{i}=\sum_{j=1}^{n} \beta_{j}\left(\tilde{\mathbf{\Omega}}^{\prime}(t)\right) h_{j i}
$$

Hence,

$$
\begin{aligned}
\lim _{t \rightarrow 0} \mathcal{I}_{i} & =\lim _{t \rightarrow 0} \sum_{j=1}^{n} \beta_{j}\left(\tilde{\boldsymbol{\Omega}}^{\prime}(t)\right) h_{j i} \\
& =\sum_{j=1}^{n}\left(\lim _{t \rightarrow 0} t \beta_{j}\left(\tilde{\boldsymbol{\Omega}}^{\prime}(t)\right)\right)\left(\lim _{t \rightarrow 0} \frac{1}{t} h_{j i}\right) .
\end{aligned}
$$

In this case,

$$
h_{j i}= \begin{cases}\frac{d_{i i}}{d_{i i}+\frac{1}{t} D_{-i}} & \text { if } i=j \\ -s_{i} \frac{1}{t}\left(\frac{t^{\lambda} k_{p}}{\sqrt{d_{j j}+\frac{1}{t} D_{-j}}}+\frac{t^{\lambda} k_{r}}{\sqrt{\frac{1}{t} d_{i j}}}\right) & \text { otherwise. }\end{cases}
$$

When $j \neq i$, we have $\lim _{t \rightarrow 0}(1 / t) h_{j i}$ if $\lim _{t \rightarrow 0} t^{-1+\lambda+1 / 2}=0$, which is true because $\lambda>1$.

Then we can write the limit of $i$ 's influence as

$$
\begin{aligned}
\lim _{t \rightarrow 0} \mathcal{I}_{i} & =\lim _{t \rightarrow 0} t \beta_{j}\left(\tilde{\mathbf{\Omega}}^{\prime}(t)\right) \lim _{t \rightarrow 0} \frac{1}{t} \frac{d_{i i}}{d_{i i}+\frac{1}{t} D_{-i}}=\lim _{t \rightarrow 0} t \beta_{j}\left(\tilde{\mathbf{\Omega}}^{\prime}(t)\right) \lim _{t \rightarrow 0} \frac{d_{i i}}{t d_{i i}+D_{-i}} \\
& =\left(\lim _{t \rightarrow 0} t \beta_{i}\left(\tilde{\boldsymbol{\Omega}}^{\prime}(t)\right)\right) \frac{d_{i i}}{D_{-i}} .
\end{aligned}
$$

We can now apply Lemma 10 to get the result:

$$
\lim _{t \rightarrow 0} \frac{\mathcal{I}_{i}(t)}{\mathcal{I}_{j}(t)}=\left(\lim _{t \rightarrow 0} \frac{t \beta_{i}\left(\tilde{\mathbf{\Omega}}^{\prime}(t)\right)}{t \beta_{i}\left(\tilde{\mathbf{\Omega}}^{\prime}(t)\right)}\right) \frac{\frac{d_{i i}}{D_{-i}}}{\frac{d_{j j}}{D_{-j}}}=\frac{\iota_{i}}{\iota_{j}} \frac{\frac{d_{i i}}{D_{-i}}}{\frac{d_{j j}}{D_{-j}}} .
$$

Step 5. Theorem 1 requires that communication costs are sufficiently low so as to have an interior solution. In the previous four steps, we operated under the assumption that nonnegativity constraints are not binding. In this last step, we vindicate that assumption by showing that when $t$ approaches 0 , we do not hit any boundary in the actions and communication intensities decisions. 
Lemma 11. If the nonnegativity constraints on $\mathbf{b}_{i}, \mathbf{r}_{-i}$, and $\mathbf{p}_{-i}$ are not binding for $t=1$, then they are not binding for any $0<t<\bar{t}$ for some $\bar{t} \in(0,1)$.

Proof. Consider

$$
\tilde{u}_{i}(a)=-\left(d_{i i}\left(a_{i}-\theta_{i}\right)^{2}+\frac{1}{t} \sum_{j \neq i} d_{i j}\left(a_{i}-a_{j}\right)^{2}+t^{\lambda} k_{r}^{2} \sum_{j \neq i} r_{j i}+t^{\lambda} k_{p}^{2} \sum_{j \neq i} p_{i j}\right) .
$$

To prove that $\mathbf{b}_{i}$ does not hit a boundary condition, just note that when $t$ tends to 0 , the vectors $\mathbf{h}_{i}(t)$ converge to a nonnegative vector for all $i$. Since if $d_{j k}>0$ for all $j \neq k$, the matrix $(\mathbf{I}-\tilde{\mathbf{\Omega}}(t))^{-1}$ is strictly positive for all $t \in(0,1)$, then the vector $(\mathbf{I}-\tilde{\boldsymbol{\Omega}}(t))^{-1} \mathbf{h}_{j}(t)$ is nonnegative if $t$ is close to 0 .

To prove interiority of active communication decisions, we can rewrite $r_{i j}$ for each $i$, $j$ with $i \neq j$ as

$$
\begin{aligned}
r_{i j}(t) & =\frac{\sqrt{d_{j i}}}{k_{r}} b_{i j} \\
& =(\mathbf{I}-\tilde{\boldsymbol{\Omega}}(t))_{i j}^{-1} \frac{\sqrt{d_{j i}}}{k_{r}} \frac{d_{j j}}{D_{j}}-s_{j} \sum_{l \neq j}(\mathbf{I}-\tilde{\boldsymbol{\Omega}}(t))_{i l}^{-1}\left(\frac{k_{p}}{k_{r}} \sqrt{\frac{d_{j i}}{D_{j}}}+1\right) \\
& =(\mathbf{I}-\tilde{\mathbf{\Omega}}(t))_{i j}^{-1}\left[\frac{\sqrt{d_{j i}}}{k_{r}} \frac{d_{j j}}{D_{j}}-s_{j} \sum_{l \neq j} \frac{(\mathbf{I}-\tilde{\boldsymbol{\Omega}}(t))_{i l}^{-1}}{(\mathbf{I}-\tilde{\mathbf{\Omega}}(t))_{i j}^{-1}}\left(\frac{k_{p}}{k_{r}} \sqrt{\frac{d_{j i}}{D_{j}}}+1\right)\right] .
\end{aligned}
$$

The first element $(\mathbf{I}-\tilde{\boldsymbol{\Omega}}(t))_{i j}^{-1}$ tends to $+\infty$ when $t$ tends to 0 . If $\lambda$ is sufficiently large, the first term in the expression in brackets is positive and tends to infinity as $t$ tends to 0 , while the second term in brackets, which is negative, is bounded below because the ratio

$$
\frac{(\mathbf{I}-\tilde{\boldsymbol{\Omega}}(t))_{i l}^{-1}}{(\mathbf{I}-\tilde{\mathbf{\Omega}}(t))_{i j}^{-1}}
$$

is positive and bounded as a consequence of Lemma 9 and the fact that $(\mathbf{I}-\tilde{\mathbf{\Omega}}(t))^{-1}$ is a nonnegative matrix, and all its entries grow at the same rate if $d_{i j}>0$ for all $i \neq j$.

Altogether, this proves that $r_{i j}(t)$ tends to $+\infty$ when $t$ tends to 0 . A similar argument applies for the case of passive communication. This finishes the proof of the theorem.

\section{REFERENCES}

Acemoglu, Daron, Munther A. Dahleh, Ilan Lobel, and Asuman Ozdaglar (2011), "Bayesian learning in social networks." Review of Economic Studies, 78, 1201-1236. [677] Akao, Yoji (1990), Quality Function Deployment: Integrating Customer Requirements Into Product Design. Productivity Press, Cambridge, Massachusetts. [668]

Alonso, Ricardo, Wouter Dessein, and Niko Matouschek (2008), "When does coordination require centralization?" American Economic Review, 98, 145-179. [653] 
Angeletos, George-Marios and Alessandro Pavan (2009), "Policy with dispersed information." Journal of the European Economic Association, 7, 11-60. [663]

Arrow, Kenneth J. (1974), The Limits of Organization. Norton, New York. [650, 654]

Bala, Venkatesh and Sanjeev Goyal (1998), "Learning from neighbours." Review of Economic Studies, 65, 595-621. [677]

Ballester, Coralio, Antoni Calvó-Armengol, and Yves Zenou (2006), "Who's who in networks. Wanted: The key player." Econometrica, 74, 1403-1417. [652, 656, 672, 680, 681]

Bandiera, Oriana, Luigi Guiso, Andrea Prat, and Raffaella Sadun (2011), "What do CEOs do?" Discussion Paper DP8235, CEPR. [650]

Bloch, Francis and Bhaskar Dutta (2009), "Communication networks with endogenous link strength.” Games and Economic Behavior, 66, 39-56. [655]

Bolton, Patrick and Mathias Dewatripont (1994), "The firm as a communication network." Quarterly Journal of Economics, 109, 809-839. [654]

Bonacich, Phillip (1987), "Power and centrality: A family of measures." American Journal of Sociology, 92, 1170-1182. [672, 680]

Bramoullé, Yann and Rachel Kranton (2007), "Public goods in networks.” Journal of Economic Theory, 135, 478-494. [656]

Cabrales, Antonio, Antoni Calvó-Armengol, and Yves Zenou (2011), "Social interactions and spillovers." Games and Economic Behavior, 72, 339-360. [656]

Calvó-Armengol, Antoni and Joan de Martí Beltran (2009), "Information gathering in organizations: Equilibrium, welfare, and optimal network structure." Journal of the European Economic Association, 7, 116-161. [654]

Crawford, Vincent P. and Joel Sobel (1982), "Strategic information transmission." Econometrica, 50, 1431-1451. [655]

Crémer, Jacques (1993), “Corporate culture and shared knowledge." Industrial and Corporate Change, 2, 351-386. [654]

Crémer, Jacques, Luis Garicano, and Andrea Prat (2007), "Language and the theory of the firm." Quarterly Journal of Economics, 122, 373-407. [654]

Dalton, Melville (1959), Men Who Manage: Fusions of Feeling and Theory in Administration. Wiley, New York. [651, 664, 670, 674, 676]

Dessein, Wouter, Andrea Galeotti, and Tano Santos (2013), "Rational inattention and organizational focus.” Discussion Paper DP9395, CEPR. [653, 677]

Dessein, Wouter and Tano Santos (2006), “Adaptive organizations.” Journal of Political Economy, 114, 956-995. [653, 664]

Dewan, Torun and David P. Myatt (2008), "The qualities of leadership: Direction, communication, and obfuscation." American Political Science Review, 102, 351-368. $[654,663]$ 
Dewatripont, Mathias and Jean Tirole (2005), "Modes of communication." Journal of Political Economy, 113, 1217-1238. [653, 664]

Dodds, Peter S., Duncan J. Watts, and Charles F. Sabel (2003), "Information exchange and the robustness of organizational networks." Proceedings of the National Academy of Sciences, 100, 12516-12521. [654]

Elliott, Matthew and Benjamin Golub (2013), "A network approach to public goods." In Proceedings of the Fourteenth ACM Conference on Electronic Commerce, 377-378, ACM, New York. [655]

Gal-Or, Esther (1985), "Information sharing in oligopoly." Econometrica, 53, 329-343. [655]

Gal-Or, Esther (1986), "Information transmission—Cournot and Bertrand equilibrium." Review of Economic Studies, 53, 85-92. [655]

Galeotti, Andrea, Christian Ghiglino, and Francesco Squintani (2013), "Strategic information transmission in networks." Journal of Economic Theory, 148, 1751-1769. [655]

Galeotti, Andrea and Sanjeev Goyal (2010), "The law of the few." American Economic Review, 100, 1468-1492. [656]

Galeotti, Andrea, Sanjeev Goyal, Matthew O. Jackson, Fernando Vega-Redondo, and Leeat Yariv (2010), “Network games.” Review of Economic Studies, 77, 218-244. [656]

Garicano, Luis (2000), "Hierarchies and the organization of knowledge in production." Journal of Political Economy, 108, 874-904. [654]

Garicano, Luis and Andrea Prat (2013), "Organizational economics with cognitive costs." In Advances in Economics and Econometrics (Daron Acemoglu, Manuel Arellano, and Eddie Dekel, eds.), 342-390, Cambridge University Press, New York. [658, 677]

Golub, Benjamin and Matthew O. Jackson (2010), "Naive learning in social networks and the wisdom of crowds." American Economic Journal: Microeconomics, 2, 112-149. [655]

Golub, Benjamin and Carlos Lever (2010), "The leverage of weak ties: How linking groups affects inequality." Working paper, Stanford University. [655, 672]

Granovetter, Mark S. (1973), "The strength of weak ties.” American Journal of Sociology, 78, 1360-1380. [650]

Griffin, Abbie and John R. Hauser (1992), "Patterns of communication among marketing, engineering, and manufacturing-A comparison between two new product teams." Management Science, 38, 360-373. [668]

Guimerà, Roger, Albert Díaz-Guilera, Fernando Vega-Redondo, Antonio Cabrales, and Alex Arenas (2002), "Optimal network topologies for local search with congestion." Physical Review Letters, 89, 248701. [654]

Hagenbach, Jeanne and Frédéric Koessler (2010), "Strategic communication networks." Review of Economic Studies, 77, 1072-1099. [655] 
Hellwig, Christian and Laura Veldkamp (2009), "Knowing what others know: Coordination motives in information acquisition.” Review of Economic Studies, 76, 223-251. [654]

Ichniowski, Casey, Kathryn Shaw, and Giovanna Prennushi (1997), "The effects of human resource management practices on productivity: A study of steel finishing lines." American Economic Review, 87, 291-313. [651, 669]

Ichniowski, Casey and Kathryn Shaw (2004), "Connective capital: Building problemsolving networks within firms.” Working paper, Stanford University. [669]

Ioannides, Yannis M. (2012), “Complexity and organizational architecture.” Mathematical Social Sciences, 64, 193-202. [654]

Jackson, Matthew O. (2008), Social and Economic Networks. Princeton University Press, Princeton, New Jersey. [672]

Jackson, Matthew O. and Asher Wolinsky (1996), "A strategic model of social and economic networks." Journal of Economic Theory, 71, 44-74. [655]

Marschak, Jacob and Roy Radner (1972), Economic Theory of Teams. Yale University Press, New Haven, Connecticut. [653, 658]

Mintzberg, Henry (1973), The Nature of Managerial Work. Harper \& Row, New York. [650]

Palacios-Huerta, Ignacio and Andrea Prat (2010), "Measuring the impact factor of agents within an organization using communication patterns." Discussion Paper DP8040, CEPR. [677]

Palacios-Huerta, Ignacio and Oscar Volij (2004), “The measurement of intellectual influence." Econometrica, 72, 963-977. [670]

Prat, Andrea (2002), "Should a team be homogeneous?" European Economic Review, 46, 1187-1207. [654]

Radner, Roy (1993), “The organization of decentralized information processing.” Econometrica, 61, 1109-1146. [654]

Raith, Michael (1996), "A general model of information sharing in oligopoly." Journal of Economic Theory, 71, 260-288. [655]

Rantakari, Heikki (2008), “Governing adaptation.” Review of Economic Studies, 75, 1257-1285. [653]

Rogers, Brian (2008), "A strategic theory of network status.” Working paper, Northwestern University. [655, 656]

Simon, Herbert A. (1986), "The impact of electronic communication on organizations." In Organizing Industrial Development (Rolf Wolff, ed.), 251-266, Walter de Gruyter, Berlin. [650]

Sobel, Joel (2013), “Giving and receiving advice.” In Advances in Economics and Econometrics: Tenth World Congress (Daron Acemoglu, Manuel Arellano, and Eddie Dekel, eds.), 305-341, Cambridge University Press, New York. [655] 
Van Zandt, Timothy (1999a), "Decentralized information processing in the theory of organizations." In Contemporary Economic Issues, Vol. 4: Economic Design and Behavior (Murat Sertel, ed.), 125-160, MacMillan Press Ltd, London. [654]

Van Zandt, Timothy (1999b), "Real-time decentralized information processing as a model of organizations with boundedly rational agents." Review of Economic Studies, 66, 633-658. [654]

Van Zandt, Timothy (2004), "Information overload in a network of targeted communication." RAND Journal of Economics, 35, 542-560. [653]

Vives, Xavier (1984), “Duopoly information equilibrium: Cournot and Bertrand.” Journal of Economic Theory, 34, 71-94. [655]

Vives, Xavier (1999), Oligopoly Pricing: Old Ideas and New Tools. MIT Press, Cambridge, Massachusetts. [655]

Weber, Max (1978), Economy and Society. University of California Press, Berkeley, California. [674]

Submitted 2013-2-22. Final version accepted 2014-6-25. Available online 2014-7-1. 